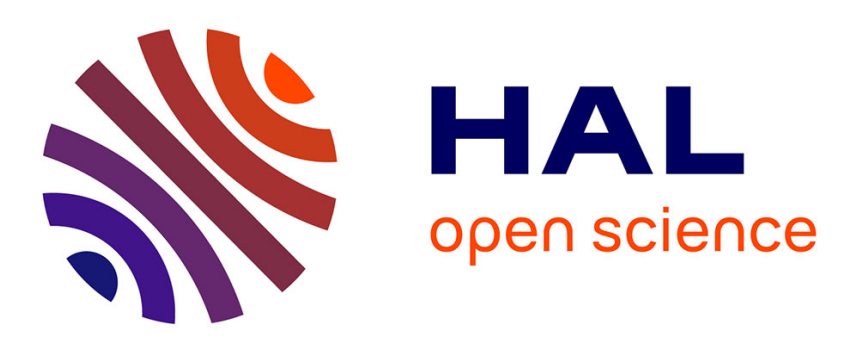

\title{
Multiscale modeling of the thermomechanical behavior in heterogeneous media embedding Phase Change Materials particles
}

Kossi-Mensah Kodjo, Julien Yvonnet, Mustapha Karkri, Karam Sab

\section{- To cite this version:}

Kossi-Mensah Kodjo, Julien Yvonnet, Mustapha Karkri, Karam Sab. Multiscale modeling of the thermomechanical behavior in heterogeneous media embedding Phase Change Materials particles. Journal of Computational Physics, 2019, 378, pp.303-323. 10.1016/j.jcp.2018.11.014 hal-02265331

\section{HAL Id: hal-02265331 \\ https://hal.science/hal-02265331}

Submitted on 9 Aug 2019

HAL is a multi-disciplinary open access archive for the deposit and dissemination of scientific research documents, whether they are published or not. The documents may come from teaching and research institutions in France or abroad, or from public or private research centers.
L'archive ouverte pluridisciplinaire HAL, est destinée au dépôt et à la diffusion de documents scientifiques de niveau recherche, publiés ou non, émanant des établissements d'enseignement et de recherche français ou étrangers, des laboratoires publics ou privés. 


\title{
Multiscale modeling of the thermomechanical behavior in heterogeneous media embedding Phase Change Materials particles
}

\author{
Kossi-Mensah Kodjo ${ }^{a, b, c}$, Julien Yvonnet ${ }^{a, *}$, Mustapha Karkrib ${ }^{\text {, Karam }}$ Sab $^{\mathrm{c}}$ \\ ${ }^{a}$ Université Paris-Est, Laboratoire Modélisation et Simulation Multi Echelle MSME UMR 8208 CNRS, 5 bd Descartes, 77454 Marne-la-Vallée, \\ France \\ ${ }^{b}$ Université Paris Est, CERTES, 61 Av. du Général de Gaulle, 94010 Créteil Cedex, France \\ ${ }^{c}$ Université Paris-Est, Laboratoire Navier, CNRS UMR 8205, ENPC, IFSTTAR, 6 et 8 avenue Blaise Pascal, 77455 Marne-la-Vallée Cedex, France
}

\begin{abstract}
In this work, a multiscale model for thermomechanical properties of composite structures containing phase change particles is developed. For the mechanical part, a classical linear computational homogenization procedure is employed. For the thermal part, due to the strong nonlinear, history-dependent thermal effects, a concurrent multiscale $\left(\mathrm{FE}^{2}\right)$ method is extended to take into account the presence of Phase Change Materials particles (PCM) at the microscale. The PCM inclusions change from liquid to solid state in the range of room temperature. This phase change induces a modified macroscopic thermal behavior, which can be used e.g. to design materials with enhanced thermal inertia and reduce energy consumption in civil engineering constructions. The technique allows taking into account accurately the fully nonlinear, history-dependent thermal behavior through numerical calculations at the microscale based on a Representative Volume Element (RVE) and its effect at the macroscale. The method is applied to concrete material including paraffin wax PCM. The results show the benefits of the PCM on the thermal behavior, including shifted and smoothed temperature response as compared to materials without PCM particles.
\end{abstract}

Keywords: Phase Change Material, Heat conduction, Computational homogenization, Multiscale modeling, $\mathrm{FE}^{2}$ method

\section{Introduction}

PCMs generally refer to substances with high latent heat, and which can change phase from liquid to solid over a restricted temperature range. These materials are able to store and release a significant amount of energy during thermal phase changes between solid and liquid states. To improve thermal inertia of buildings and reduce energy consumption, several works have demonstrated the effectiveness of building materials embedding PCMs [1, 2, 3, 4, 5]. For example in [6], different paraffin samples have been tested and defined as the most advantageous PCMs for building applications. In passive applications of solar energy, paraffin can be introduced into building materials to provide energy storage around the comfort temperature of $25^{\circ} \mathrm{C}$. PCM wall can be constructed by incorporating PCMs into construction materials such as cement, concrete and bricks (see Fig. 1). The incorporated PCMs can increase the thermal inertia of the wall so as to reduce the indoor temperature fluctuations, to increase the thermal comfort and to reduce the energy consumption due to air conditioning or heating.

The use of PCM composites in buildings is still in experimental stage, and requires simulation methodologies to predict and design these new structures. The present limitations are related to the strong nonlinear, history-dependent

\section{$* *$}

Email address: julien.yvonnet@univ-paris-est.fr (Julien Yvonnet) 


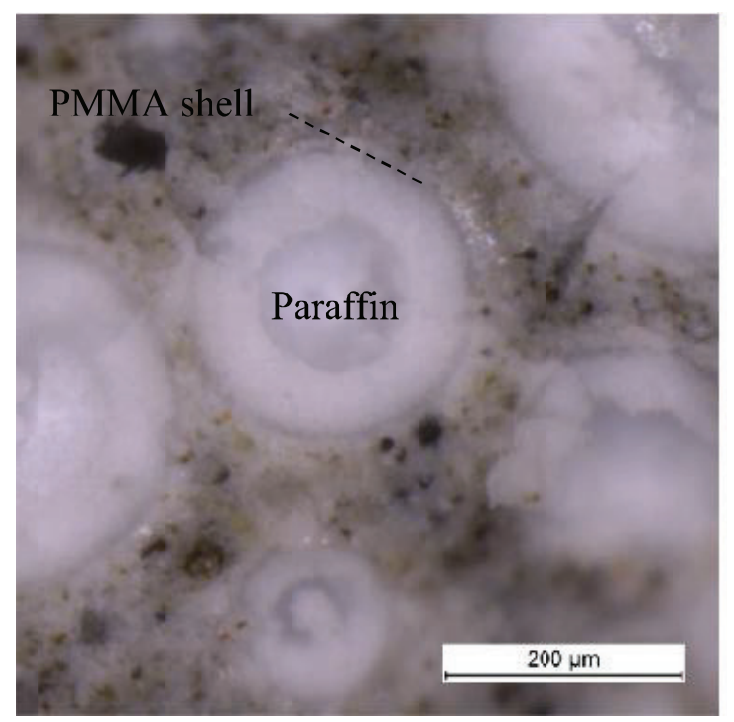

Figure 1: Micrographs of encapsulated PCM (Micronal DS 5040 X) embedded in cement-paste matrix [7]

thermomechanical behavior of such materials and its multiscale nature. Due to the nonlinear phase change at the microscale, classical thermal models cannot be employed at the macroscale, and defining the overall macroscopic behavior cannot be performed by classical (linear) homogenization approaches. In addition, describing explicitly all heterogeneities in a numerical nonlinear model such as the Finite Element Method is not feasible due to the scale separation, i.e. the large ratio between the characteristic dimensions of the structures and of the PCM capsules.

In [8], an algorithm based on Finite Volume Method was employed to simulate a 2D model of PCMs incorporated in porous metals, combining fluid dynamics and heat transfer. In [9], a Volume Of Fluid model was used to simulate thermal phase changes. In [10], an analytical model based on Mori-Tanaka method [11] was developed to evaluate thermal performances of composites including PCMs, but neglecting phase changes effects.

In the present work, we propose to model the fully nonlinear thermomechanical behavior of PCM composites by using a concurrent multiscale method ( $\mathrm{FE}^{2}$ method, see e.g. $[12,13,14,15]$ and a recent review on these classes of methods in [16]). The $\mathrm{FE}^{2}$ employs at each integration point of the macroscopic finite element structural model an explicit description of the representative Volume Element, whose nonlinear response is obtained numerically and transferred to the structure through boundary conditions and numerical averaging operations. The technique has recently been applied to nonlinear conduction (without phase changes) in [17, 18]. The objective of this work is to extend the $\mathrm{FE}^{2}$ method to RVE containing PCM, where local equations include fluid mechanics combined with thermal phase change and description of solid/liquid transitions. The overall response of the structure can then be obtained numerically to describe the effects of the PCM on the response of the structure, which is proposed here for the first time, to our best knowledge.

The overview of the present paper is as follows: in section 2, the equations at the microscale are presented, describing the liquid-solid phase change. In section 3, the mechanical macroscopic model is described, and we show that in the assumption of weak thermomechanical coupling, these effective properties can be computed apart from thermal ones. The coupling between the micro and macroscale nonlinear thermal properties is provided in section 4. Finally, 2D and 3D numerical examples are presented in section 5 to verify the method and to study the effects of PCM on the overall thermal response of the structure.

\section{Microscale phase change thermal model}

In this section, we present the equations of the model to be solved at the microscale, involving the presence of a fluid in capsules which can undergo phase change. We consider the RVE described in Fig. 2. The matrix is assumed 


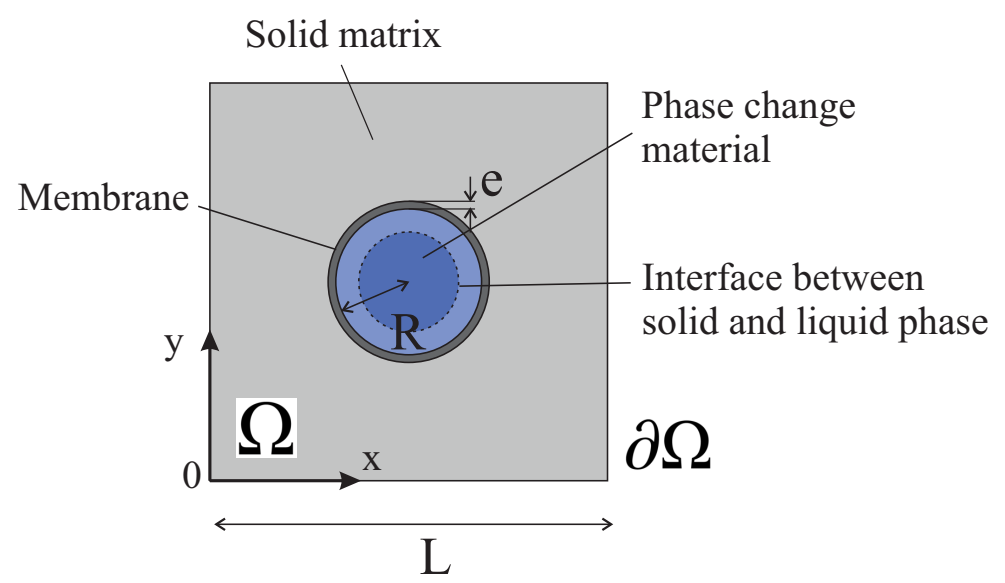

(a)

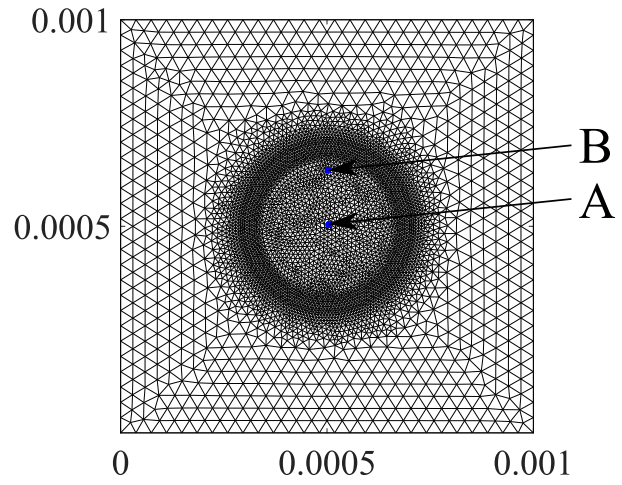

(b)

Figure 2: RVE associated with the microscale embedding phase change inclusion: (a) geometry; (b) Finite Element Mesh.

to be linearly elastic. The thermal model for the matrix is assumed to be linear. The capsule is composed of a membrane, considered as a linear solid with finite thickness. Inside the capsule, the PCM can change from solid to liquid according to the temperature. The related equations are provided in the following.

\subsection{Equations describing the PCM}

To model the phase change and the thermal behavior within the PCM, the equivalent heat capacity method is used [19]. In its liquid phase, the PCM is considered as an incompressible Newtonian fluid. The interfaces are assumed to be perfect and viscous dissipation is neglected. In addition, we neglect the volume change associated with phase transition. Under these assumptions, the governing equations are provided as follows. In the matrix, the membrane and the solid phase of the PCM, the energy conservation is expressed as:

$$
\rho_{s} C_{p s} \frac{\partial T}{\partial t}=\nabla \cdot\left(\lambda_{s} \nabla T\right)
$$

where $\nabla \cdot($.$) denotes the divergence operator, \rho_{s}$ is the density, $C_{p s}$ the specific heat, $T$ the temperature, $t$ the time, $\lambda_{s}$ the thermal conductivity matrix. The conduction is assumed to be isotropic, i.e. $\lambda_{s}=\lambda_{s} \mathbf{1}$, and $\mathbf{1}$ is the second-order identity tensor. The mass conservation within the liquid phase of the PCM is given by:

$$
\nabla \cdot \mathbf{v}=0
$$

where $\mathbf{v}$ is the fluid velocity. The equilibrium is given by:

$$
\rho_{\ell} \frac{\partial \mathbf{v}}{\partial t}+\rho_{\ell}(\mathbf{v} \cdot \nabla \mathbf{v})=\nabla \cdot\left[-p \mathbf{1}+\eta_{a}\left(\nabla \mathbf{v}+(\nabla \mathbf{v})^{T}\right)\right]+\mathbf{F}_{b}
$$

where $\rho_{\ell}$ is density of the liquid PCM, $p$ is the pressure, $\eta_{a}$ is the dynamic viscosity, $\nabla($.$) is the gradient operator and$ $\mathbf{F}_{b}$ is used to force a trivial solution of $\mathbf{v}=0$ in the solid PCM:

$$
\mathbf{F}_{b}=-A_{v}(T) \mathbf{v}
$$

According to the Darcy model used in [20], $A_{v}(T)$ is given by

$$
A(T)=C \frac{(1-f(T))^{2}}{(f(T))^{3}+b},
$$

where $b=10^{-3}$ is a numerical perturbation parameter which prevents from divisions by zero, and $C$ is another constant whose numerical values range between $10^{4}$ and $10^{7}$, and acts on the melting front. In [21] for example, $C=10^{5}$ was chosen. The liquid fraction $f$ in the PCM is defined by: 


$$
f(T)=\left\{\begin{array}{l}
0 \text { if } T<T_{s}, \\
\frac{T-T_{s}}{T_{\ell}-T_{s}} \text { if } T_{s}<T<T_{\ell}, \\
1 \text { if } T>T_{\ell},
\end{array}\right.
$$

where $T_{s}$ is the temperature transformation from solid to liquid and $T_{\ell}$ is the temperature at which the transformation from liquid to solid begins. Below $T_{s}$, the PCM is solid and above $T_{\ell}$, the PCM is liquid. Between $T_{s}$ and $T_{\ell}$, there is a smooth description of the phase transition, where $\lambda, \eta$ and $C_{p}$ vary in the PCM. A function $\lambda_{a}(T)$ is used to take into account the variations of thermal conductivity in the PCM:

$$
\lambda_{a}(T)=\lambda_{s}+\left(\lambda_{\ell}-\lambda_{s}\right) f(T) .
$$

The following approximation is used for the apparent viscosity:

$$
\eta_{a}(T)=\eta_{\ell}\left(1+A_{v}(T)\right) .
$$

Above, $A_{v}(T)$ tends towards infinity when the PCM is solid and $\eta_{a}(T)=\eta_{l}$ when the PCM is liquid. The latent heat of fusion $L_{F}$, due to phase changes is introduced in the apparent specific heat $C_{p a}$ :

$$
C_{p a}=\left\{\begin{array}{l}
C_{p s} \text { if } T<T_{s}, \\
\frac{1}{2}\left(C_{p s}+C_{p \ell}\right)+\frac{L_{F}}{T_{\ell}-T_{s}} \text { if } T_{s}<T<T_{\ell}, \\
C_{p \ell} \text { if } T>T_{\ell} .
\end{array}\right.
$$

In (9), the apparent specific heat exhibits a peak in the temperature range between $T_{s}$ and $T_{\ell}$. This peak increases with $L_{F}$. Finally, the energy conservation in the PCM inclusion is given by [19]:

$$
\rho_{\ell} C_{p a} \frac{\partial T}{\partial t}+\rho_{\ell} C_{p a} \mathbf{v} \cdot \nabla T=\nabla \cdot\left(\lambda_{a} \nabla T\right) .
$$

We assume that the velocity of the liquid PCM on the boundary of the membrane $\Gamma_{i n c}$ is equal to zero:

$$
\mathbf{v}=\mathbf{0} \quad \text { on } \quad \Gamma_{\text {inc }} .
$$

The above equations are solved by the finite element method (see $[22,23]$ for more details). The associated mesh for FEM resolution is depicted in Fig. 2 (b).

\subsection{Illustration of PCM's thermal behavior}

In what follows, we provide a numerical illustration of the phase change within the inclusion during a temperature transition. The length of the RVE is $L=1 \mathrm{~mm}$, the radius of the PCM is $R=0.2 \mathrm{~mm}$ and the thickness of the membrane is $e=0.015 \mathrm{~mm}$ (see Fig. 2). To obtain phase transition, the temperature profile shown in Fig. 3 is prescribed on the boundaries $y=1 \mathrm{~mm}$ and $y=0 \mathrm{~mm}$, the other boundaries are insulated. The PCM has a melting point at $300.15 \mathrm{~K}$ and its latent heat of fusion is $L_{F}=1.68 \times 10^{5} \mathrm{~J}_{\mathrm{kg}}{ }^{-1}$. The dynamic viscosity of the liquid phase is $\eta_{\ell}=3.42 \times 10^{-3}$ Pa.s. The thermo-physical coefficients of each constituents are provided in Table 2 .

The prescribed temperature is increased from $293.15 \mathrm{~K}$ to $310.15 \mathrm{~K}$ in $3.5 \mathrm{~s}$, then decreased to $293.15 \mathrm{~K}$ again in 3.5 s. The phase transitions are shown in Figs 4 and 5, where the function $f(T)$ indicates the local concentration of liquid phase (in red).

In a second example, the melting process and the thermo-physical properties of the PCM are studied. Using the same RVE as previously, a constant temperature of $303.15 \mathrm{~K}$ is applied on the upper and lower sides $y=0$ and $y=L$, the heat flux is zero on the other boundaries. The temperature profiles are plotted for a point on the boundary, and in points $\mathrm{A}$ and $\mathrm{B}$, where $\mathrm{A}$ is located at the center of the domain and $\mathrm{B}$ is at $x_{B}=0.5 \mathrm{~mm}, y_{B}=0.66 \mathrm{~mm}$ (see Fig. 2 (b)). 


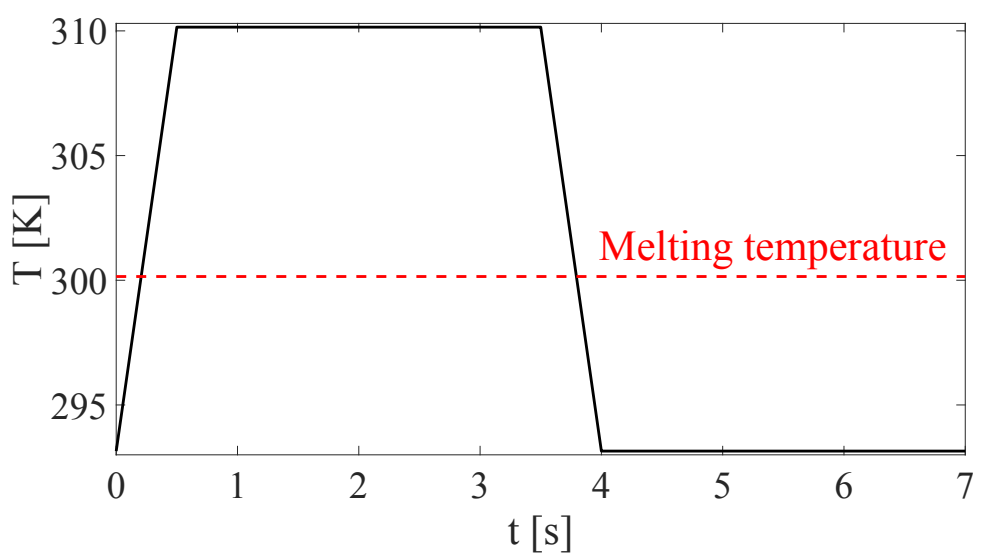

Figure 3: Temperature prescribed on the boundary of the RVE.

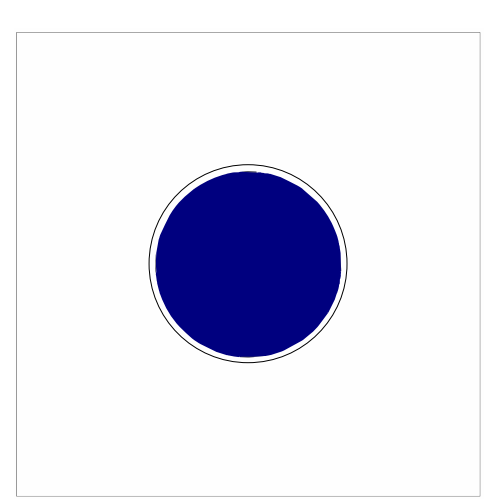

(a) $\mathrm{t}=0 \mathrm{~s}$

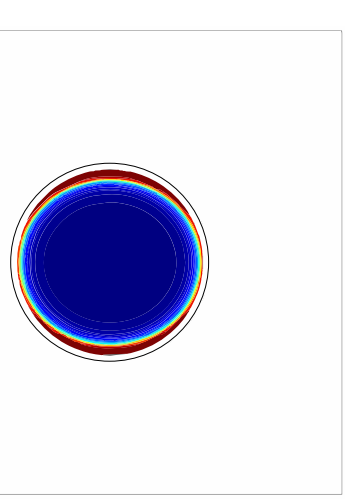

(b) $\mathrm{t}=0.75 \mathrm{~s}$

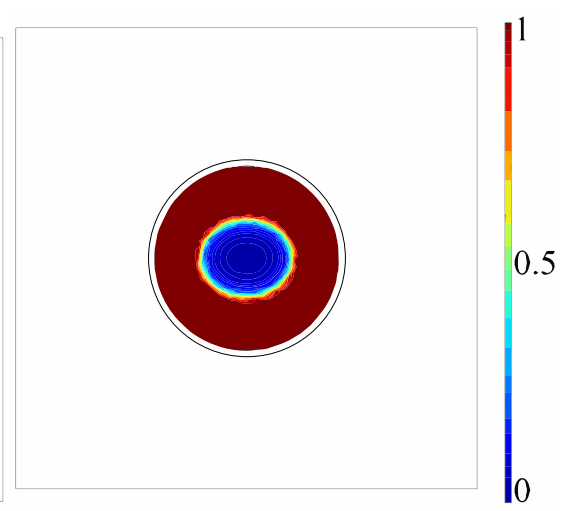

(c) $t=2 \mathrm{~s}$

Figure 4: $f(T)$ during melting $(0 \mathrm{~s}<t<3.5 \mathrm{~s})$. The red region indicates the liquid phase, the blue region denotes the solid phase.

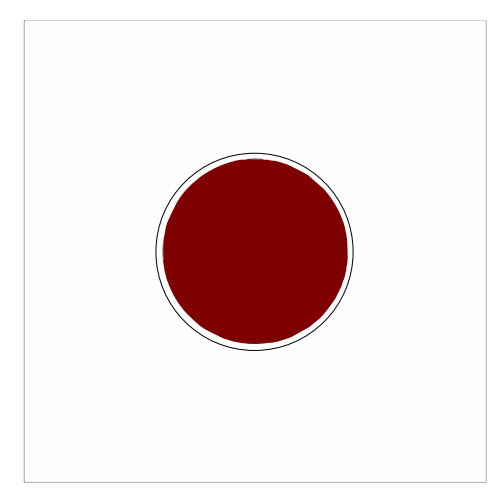

(a) $t=3.5 \mathrm{~s}$

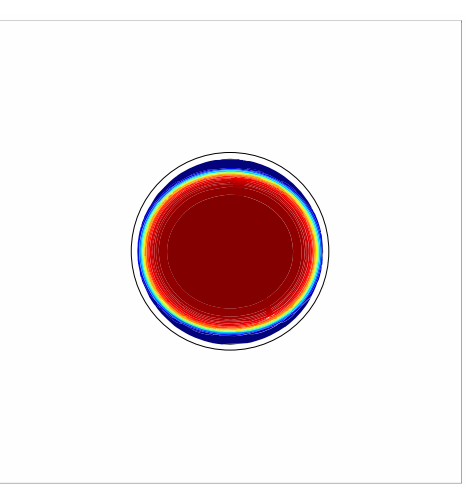

(b) $\mathrm{t}=4.5 \mathrm{~s}$

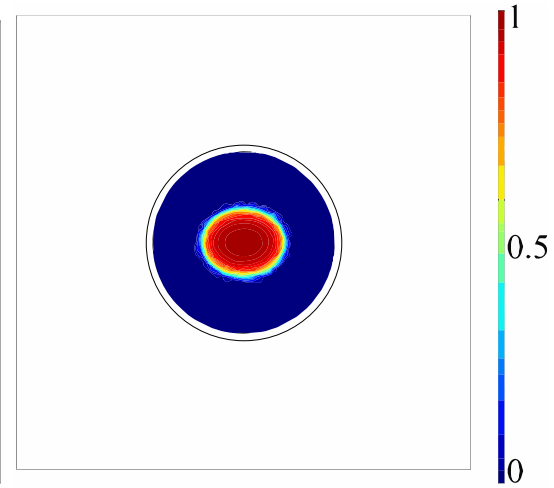

(c) $\mathrm{t}=6.0 \mathrm{~s}$

Figure 5: $f(T)$ during solidification $(3.5 \mathrm{~s}<t<7 \mathrm{~s})$. The red region indicates the liquid phase, the blue region denotes the solid phase. 


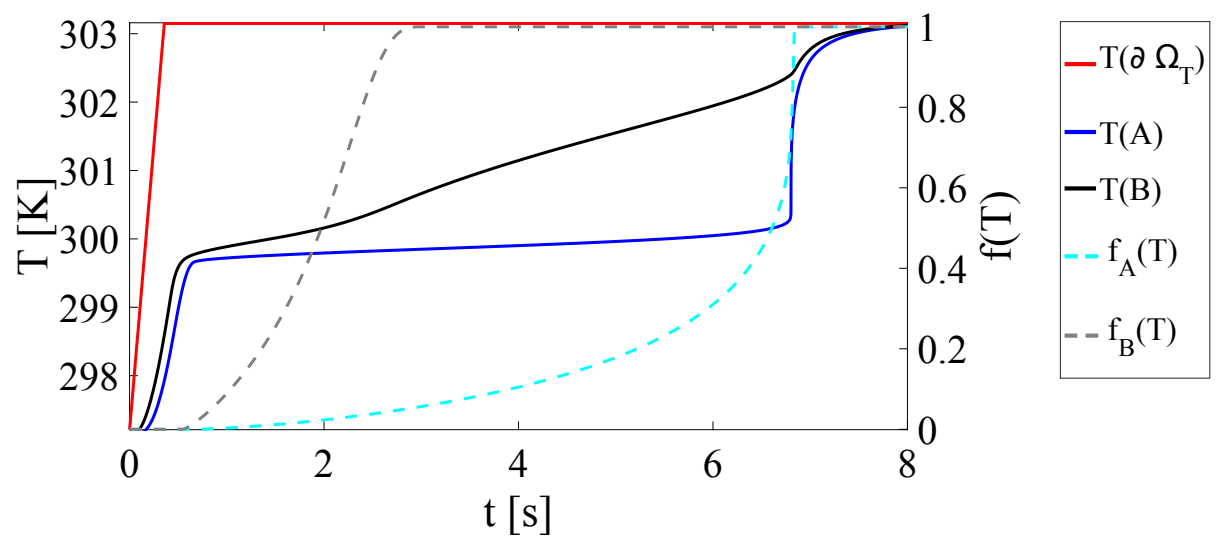

Figure 6: Temperature and liquid PCM phase indicator $f(T)$ during melting

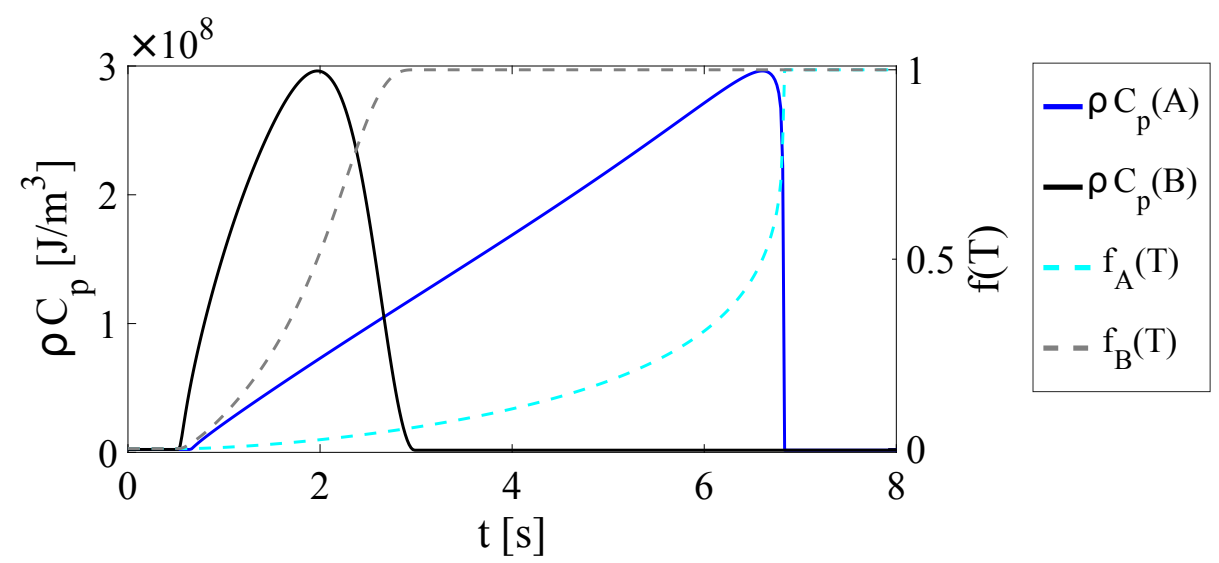

Figure 7: Variations of $\rho C_{p a}$ in the PCM during melting

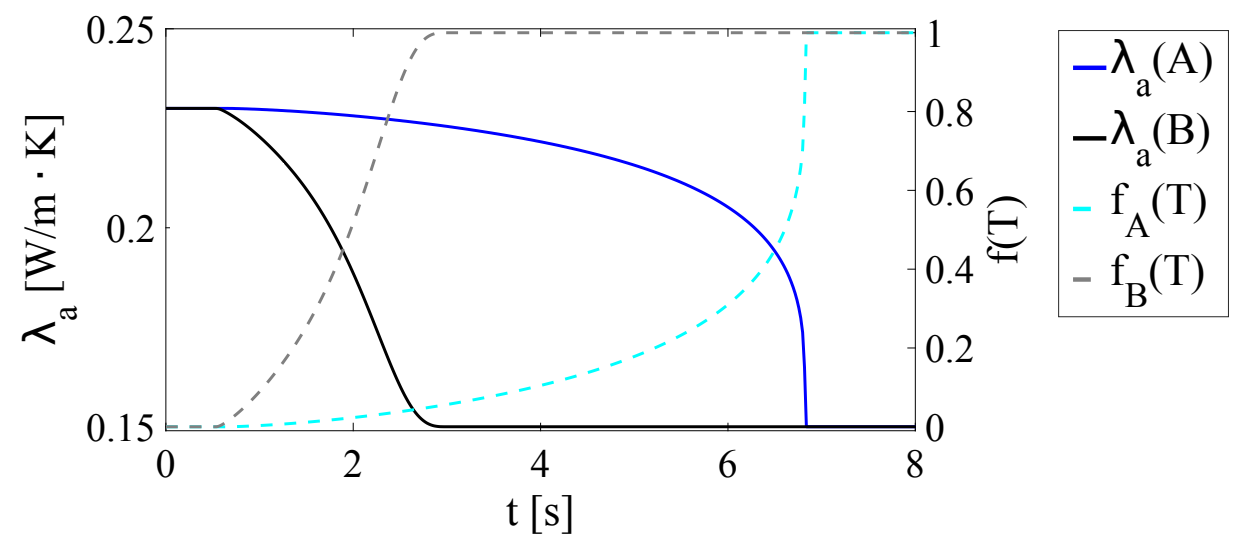

Figure 8: Variations of $\lambda_{a}$ in the PCM during melting 


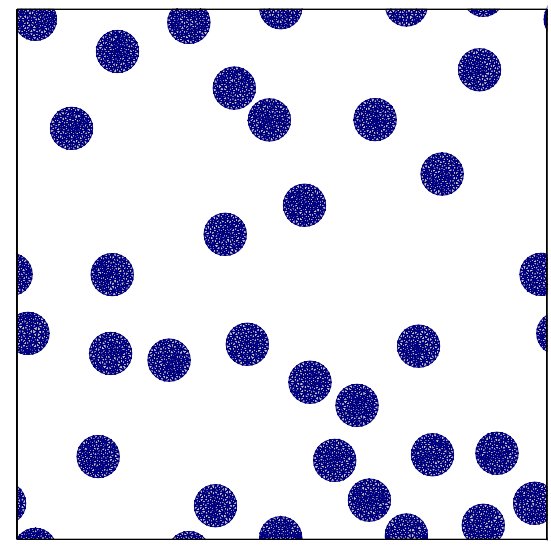

(a)

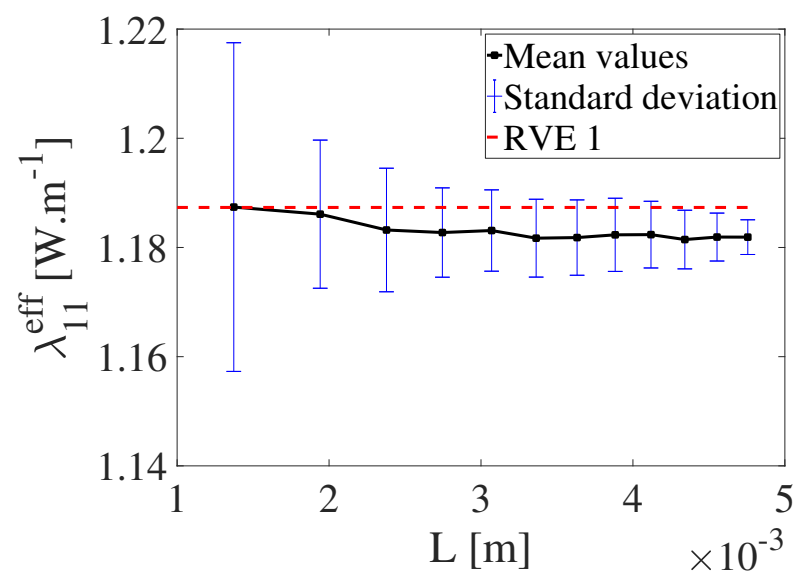

(b)

Figure 9: RVE size problem: variation of effective thermal conductivity as a function of RVE size for $20 \%$ of PCM volume fraction: (a) example of periodic microstructure with ramdomly distributed PCM particles $\left(L=5.013 \times 10^{-3} \mathrm{~m}\right.$, only the mesh of the PCM domain is displayed); (b) mean values and standard deviation of effective thermal conductivity (the red curve denote the response of the microstructure with only one PCM particle).

Phase change occurs at almost constant temperature $\left(T_{\ell}-T_{s}=1 \mathrm{~K}\right)$ as shown in Fig. 6 . The thermal properties of the PCM depend on the temperature field (see Fig. 7 and 8). This leads to a time-dependency of the effective thermo-physical properties.

There is a peak of stored energy during phase transition (solid to liquid here) due to the latent heat. This property is necessary to store and release more thermal energy in the composite. The apparent thermal conductivity at each position inside the PCM varies linearly with the liquid fraction indicator $f(T)$ as expressed by (6). The microscale parameters (energy, thermal conductivity, heat flux) which will be used at the macroscale must be computed at each time step and for every integration point. The variation of these parameters at microscale is the source of non linearities in the macroscale model. Assuming small RVE dimensions as compared to the structure, steady state conditions can be considered in the RVE. Under this assumption, Eqs. (1), (3) and (10) can be safely reduced to:

$$
\begin{gathered}
\nabla \cdot\left(\lambda_{s} \nabla T\right)=0, \\
\rho_{l}(\mathbf{v} \cdot \nabla \mathbf{v})=\nabla \cdot\left[-p \mathbf{1}+\eta_{a}\left(\nabla \mathbf{v}+(\nabla \mathbf{v})^{T}\right)\right]+\mathbf{F}_{b}, \\
\rho_{\ell} C_{p a} \mathbf{v} \cdot \nabla T=\nabla \cdot\left(\lambda_{a} \nabla T\right) .
\end{gathered}
$$

It is important to ensure that the microscale domain is representative. For this purpose, an RVE size study is conducted. The effective thermal conductivity of a composite with $20 \%$ of PCM volume fraction is considered. Different unit cells containing randomly distributed PCM particles are investigated with varying size $L$. For each size $L, 20$ realizations are generated (see Fig. 9). The thermal conductivity of the matrix is $1.6 \mathrm{~W} \cdot \mathrm{m}^{-2} \cdot \mathrm{K}^{-1}$. The PCM is assumed to be in its solid state and its thermal conductivity is $0.23 \mathrm{~W} \cdot \mathrm{m}^{-2} \cdot \mathrm{K}^{-1}$. Fig. 9 shows that the RVE containing only one inclusion gives an effective thermal conductivity with an error of $0.76 \%$ as compared to the converged mean value. Then, in the following, the RVE with only one PCM inclusion is considered in the multiscale analysis.

\section{Homogenization of mechanical properties during phase changes}

\subsection{Homogenization problem}

In this section, we investigate how the thermal phase change in the PCM affects the effective mechanical properties of the RVE. As depicted in Fig. 2, the RVE is composed of three materials: (a) the matrix, composed of solid cement 
paste; (b) the solid membrane made of PMMA; (c) the inclusion, made of paraffin wax, which can be either liquid or solid.

The numerical model presented at Section 2 is used to handle the thermal behavior of the RVE. Thus, the effective mechanical constitutive law (the elasticity tensor $\overline{\mathbb{C}}_{i j k l}$ ) is determined at each time-step of the thermal phase change by homogenization. The problem is to find the effective elastic properties of the equivalent homogeneous material while the PCM is melting or solidifying. The matrix and the membrane are assumed to have constant elastic properties, while the PCM has two phases described by $f(T)$ (liquid phase volume fraction). The phase change model gives a smooth concentration function $f(T)$ (see Fig. 6) which varies from 0 to 1 around the interface (see Figs. 4-5). Different mechanical properties are used in each phase of the PCM. The fourth-order elastic tensor $\mathbb{C}_{i j k l}$ describes the local elastic constitutive law in the matrix such that $\sigma=\mathbb{C}: \varepsilon$, with $\sigma$ and $\varepsilon$ the Cauchy stress tensor and the linearized elastic strain tensor, respectively. In order to use the smooth function $f(T)$ to define the solid-liquid interface, the liquid part of the PCM is assumed to be a linear elastic isotropic material that mimics the behavior of a static liquid, with very low shear coefficient and Poisson's ratio close to 0.5 . In summary, the stiffness matrix used for the PCM is given by:

$$
\mathbb{C}_{P C M}=(1-f(T)) \mathbb{C}_{P C M}^{S}+f(T) \mathbb{C}_{P C M}^{\ell},
$$

where $\mathbb{C}_{P C M}^{S}$ and $\mathbb{C}_{P C M}^{\ell}$ denote the stiffness matrix for the solid and liquid phase in the PCM, respectively. Assuming small strain elastostatics and neglecting body forces due to the small size of the inclusions, the problem to be solved on the RVE is given by:

$$
\nabla \cdot \sigma(\mathbf{x})=0 \forall \mathbf{x} \in \Omega
$$

with

$$
\begin{gathered}
\sigma(\mathbf{x})=\mathbb{C}(\mathbf{x}): \boldsymbol{\varepsilon}(\mathbf{x}), \\
\boldsymbol{\varepsilon}(\mathbf{x})=\frac{1}{2}\left(\nabla \mathbf{u}(\mathbf{x})+\nabla^{T} \mathbf{u}(\mathbf{x})\right)
\end{gathered}
$$

and

$$
\frac{1}{|\Omega|} \int_{\Omega} \boldsymbol{\varepsilon} \quad d \Omega=\bar{\varepsilon}
$$

In the above, $\mathbf{u}$ is the displacement field and $\bar{\varepsilon}$ is the macroscopic strain applied on the RVE. Different boundary conditions can be applied on the RVE: KUBC (Kinematic Uniform Boundary Conditions), SUBC (Static Uniform Boundary Conditions) or PB (Periodic boundary conditions). In this work, periodic boundary conditions are employed, expressed by:

$$
\mathbf{u}(\mathbf{x})=\bar{\varepsilon} \mathbf{x}+\tilde{\mathbf{u}}(\mathbf{x}),
$$

where $\tilde{\mathbf{u}}(\mathbf{x})$ is a periodic function over $\Omega$. Condition (20) can be re-written as:

$$
\mathbf{u}^{+}-\mathbf{u}^{-}=\bar{\varepsilon}\left(\mathbf{x}^{+}-\mathbf{x}^{-}\right),
$$

where $\mathbf{u}^{+}, \mathbf{u}^{-}$and $\mathbf{x}^{+}, \mathbf{x}^{-}$are respectively the displacement and the position of each pair of opposite nodes on $\partial \Omega$ (see Fig. 10). Such conditions can be prescribed e.g. by Lagrange multipliers techniques.

The problem being linear, the solution $\boldsymbol{u}$ depends on the three independent components of $\overline{\boldsymbol{\varepsilon}}$ in the $2 \mathrm{D}$ case. Using the superposition principle, we can write:

$$
\varepsilon=\varepsilon^{11} \bar{\varepsilon}_{11}+\varepsilon^{22} \bar{\varepsilon}_{22}+2 \varepsilon^{12} \bar{\varepsilon}_{12}
$$

where $\varepsilon$ is the solution of the problem (16)-(19) for an applied macroscopic strain tensor

$$
\boldsymbol{\varepsilon}^{k l}=\frac{1}{2}\left(\mathbf{e}_{k} \otimes \mathbf{e}_{l}+\mathbf{e}_{l} \otimes \mathbf{e}_{k}\right),
$$




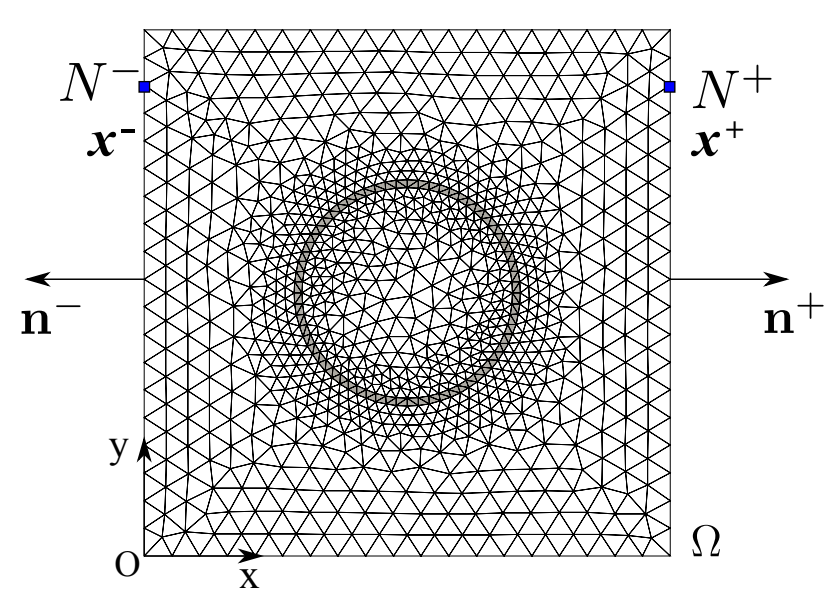

Figure 10: 2D RVE with symmetric mesh and nodes for prescribing periodic boundary conditions.

Table 1: Mechanical proprieties of the RVE

\begin{tabular}{lcc}
\hline & Young's modulus [MPa] & Poisson's ratio \\
\hline Matrix & $2 \times 10^{4}$ & 0.2 \\
\hline Membrane & $3.2 \times 10^{3}$ & 0.3 \\
\hline Solid PCM & 60 & 0.4 \\
\hline
\end{tabular}

and $\mathbf{e}_{i}, i=1,2$ in $2 \mathrm{D}$ are unitary basis vectors. The above relation can be re-written as

$$
\varepsilon(\mathbf{x})=\mathbb{A}(\mathbf{x}): \bar{\varepsilon}
$$

where the fourth-order localization tensor $\mathbb{A}$ is defined by:

$$
\mathbb{A}_{i j k l}=\boldsymbol{\varepsilon}_{i j}^{k l}
$$

Taking the space average of (17) over $\Omega$, we obtain the effective elastic tensor at each time step of thermal load:

$$
\overline{\mathbb{C}}(t)=\frac{1}{|\Omega|} \int_{\Omega} \mathbb{C}(x, t): \mathbb{A}(x, t) \quad d \Omega .
$$

\subsection{Results}

The mechanical properties are listed in the table 1. When the PCM is in the liquid phase, a pseudo elastic model with low shear modulus taken as $1 \mathrm{MPa}$ (negligible as compared to the stiffness of the matrix) and Poisson's ratio of 0.49 is considered.

The 2D RVE of Fig. 15a is used. The time-dependent temperature given in Fig. 3 is applied at $y=0$ and $y=1$ $\mathrm{mm}$ while the other boundaries are insulated. Fig. 11 shows the variation of the components of $\overline{\mathbb{C}}$ with time. All components of $\overline{\mathbb{C}}$ have similar evolutions. The unsymmetric evolution of the curves is explained as follows. During melting, the solid PCM is surrounded by the liquid phase, such that the macroscopic loads $\bar{\varepsilon}$ applied on $\partial \Omega$ cannot be transmitted to the solid part of the inclusion. But during solidification, the solid part of the PCM is connected to the shell and the matrix, so the solid phase contributes to the RVE's effective mechanical response. As expected, the variation of each component of $\overline{\mathbb{C}}$ during thermal phase changes remains small (does not exceed $1.6 \%$ in this case), because of the high ratio between the mechanical properties of the matrix and the PCM.

To confirm these results for larger volume fractions, we have conducted in Fig. 12 an analysis to evaluate the effects of a real incompressible fluid on the mechanical effective behavior. We consider two cases: (a) the present 


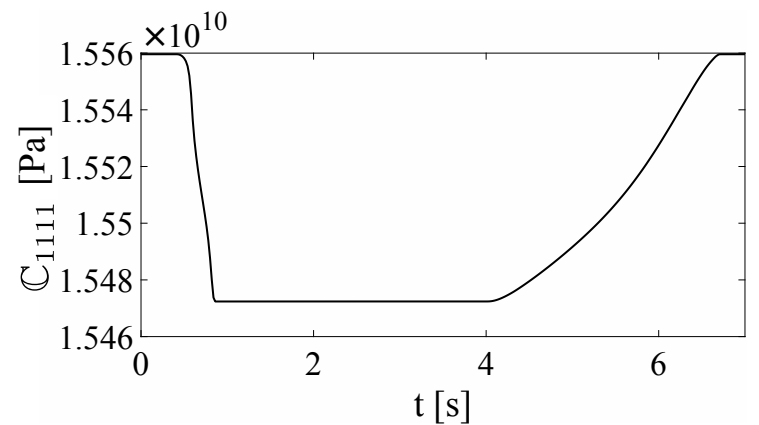

(a) $\mathbb{C}_{1111}$

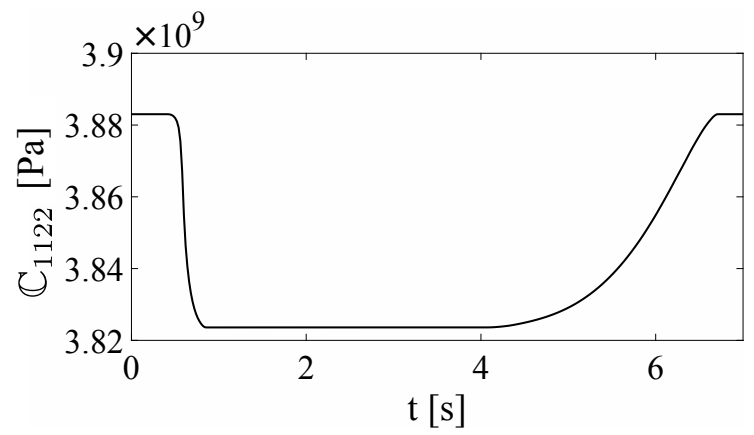

(c) $\mathbb{C}_{1122}$

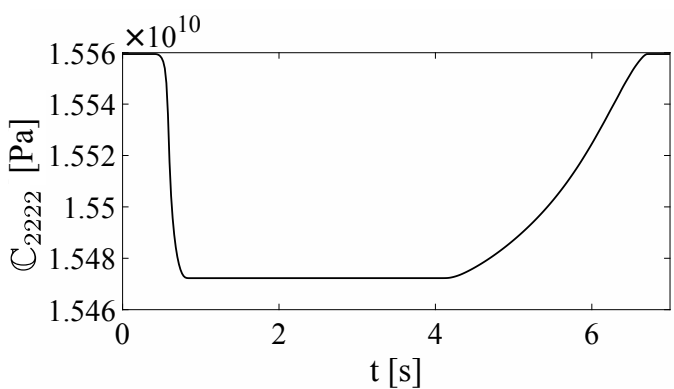

(b) $\mathbb{C}_{2222}$

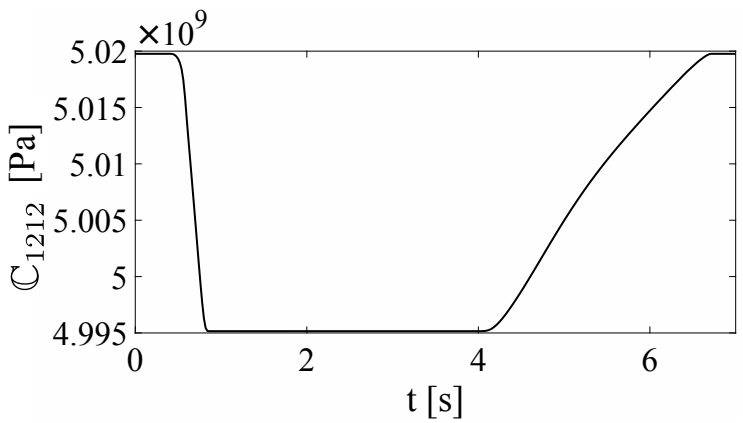

(d) $\mathbb{C}_{1212}$

Figure 11: Variation of the components of $\mathbb{C}$ during thermal phase changes.

simplified model, i.e. an RVE composed of a solid matrix and a solid PCM, whose effective properties are computed using classical linear homogenization (denoted as $\overline{\mathbb{C}}^{S}$ ), and (b) a liquid PCM where the equilibrium of the RVE for an applied strain is computed by solving a full fluid-structure interaction problem. As the details of such calculations are out of the scope of this paper, we do refer to [24] for more details. In this second case, the effective elastic properties are denoted as $\overline{\mathbb{C}}^{S-\ell}$. The relative error between the effective tensor for solid PCM $\overline{\mathbb{C}}^{S}$ and for liquid PCM is computed here for a large volume fraction of PCM (19\%) as:

$$
E_{r}=\frac{\overline{\mathbb{C}}_{i j k l}^{S}-\overline{\mathbb{C}}_{i j k l}^{S-\ell}}{\overline{\mathbb{C}}_{i j k l}^{S-\ell}} .
$$

As an example, Fig. 12 shows the relative error for the component $\overline{\mathbb{C}}_{1212}$. We can see from this curve that the present simplified model and the associated assumption of decoupled mechanical and thermal problems is valid as soon as the ratio between the Young modulus of the matrix $\left(\mathrm{E}_{m}\right)$ and of the solid PCM $\left(\mathrm{E}_{P C M}\right)$ is larger than $10^{2}$.

In conclusion, the effective elastic tensor $\overline{\mathbb{C}}$ can be assumed as constant and be computed a priori with respect to the thermal analysis, which greatly simplifies the multiscale analysis described in the next section.

\section{Multiscale $\left(\mathrm{FE}^{2}\right)$ modeling of the transient thermal behavior with phase change}

Due to the phase change of the PCM capsules, the effective thermal behavior is strongly nonlinear and the classical computational homogenization can no more be applied in that case. In this work, the $\mathrm{FE}^{2}$ method is employed and extended to transient thermal behavior with phase change to obtain at each point (integration point of the macroscopic mesh) of the structure the macroscopic thermal behavior through RVE calculations. The phase change model 


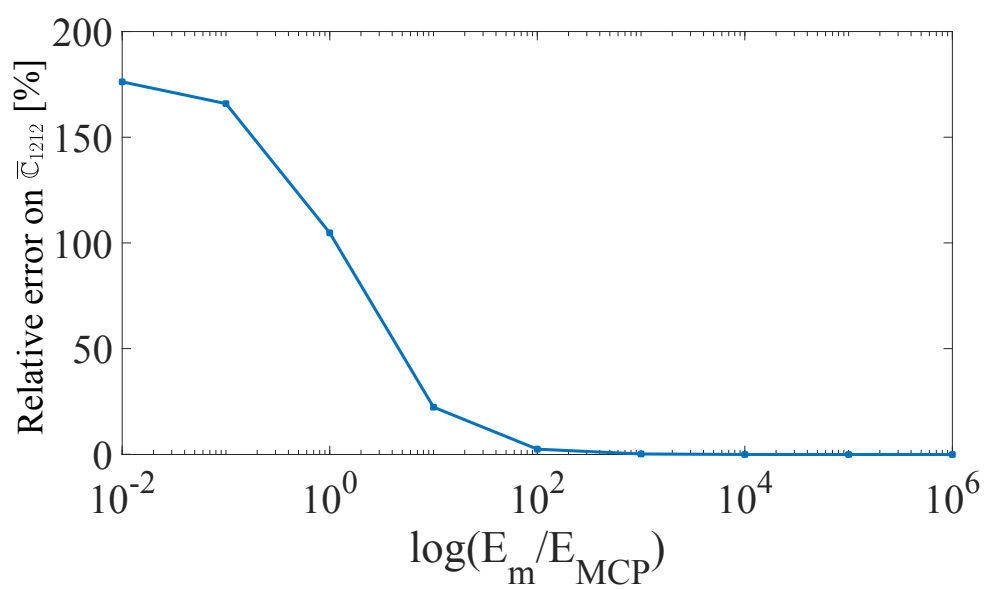

Figure 12: Relative error between $\overline{\mathbb{C}}_{1212}^{S}$ and $\overline{\mathbb{C}}_{1212}^{S-\ell}$ as a function of stiffness ratios $E_{m} / E_{P C M}$ for $19 \%$ of PCM volume fraction.

presented in section 2 is used for the microscale thermal problem. Assuming that the thermal solution has negligible impact on the mechanical solution (see section 3), the effective elastic tensor is computed separately before the analysis. Then the following $\mathrm{FE}^{2}$ procedure is only related to the nonlinear thermal analysis.

\subsection{Microscale problem}

The microscale problem is solved at each integration point of the macroscale structure (see Fig. 13) to obtain the macroscopic heat flux $\overline{\boldsymbol{\Phi}}$, the effective conductivity $\bar{\lambda}$ and the effective specific heat density $\overline{\rho C_{p}}$, given the macroscale temperature $\bar{T}$ and temperature gradient $\overline{\nabla T}$. The scale separation is assumed, which means that the characteristic size of the inclusions is much smaller than the dimensions of the structure. In addition to the local equations for the nonlinear microscopic thermal problem (see section 2), the RVE is subjected to homogeneous temperature gradient through the condition:

$$
\frac{1}{|\Omega|} \int_{\Omega} \nabla T d \Omega=\overline{\nabla T}
$$

Classically, this condition can be satisfied for the boundary conditions:

$$
T(\mathbf{x})=\overline{\nabla T} \cdot \mathbf{x} \text { on } \partial \Omega
$$

or

$$
T(\mathbf{x})=\overline{\nabla T} \cdot \mathbf{x}+\tilde{T}(\mathbf{x}) \text { on } \partial \Omega
$$

where $\tilde{T}(\mathbf{x})$ is a periodic function over $\Omega_{m}$. The boundary condition (30) can be rewritten as:

$$
T^{+}-T^{-}=\overline{\nabla T} \cdot\left(x^{+}-x^{-}\right),
$$

where $T^{+}$and $T^{-}$are the temperature at each pair of opposite nodes (see Fig. 10). To ensure a unique solution at the microscale, the macroscale temperature $\bar{T}$ is used to introduce an additional constraint such that [17]:

$$
\overline{\rho C_{p} T}=\frac{1}{\Omega} \int_{\Omega} \rho C_{p} T d \Omega
$$

\subsection{Macroscale problem}

\subsubsection{Homogenized quantities}

The macroscopic heat storage to be used is defined as its volume average over the RVE, as proposed in [17]:

$$
\overline{\rho C_{p}}=\frac{1}{|\Omega|} \int_{\Omega} \rho C_{p} d \Omega,
$$




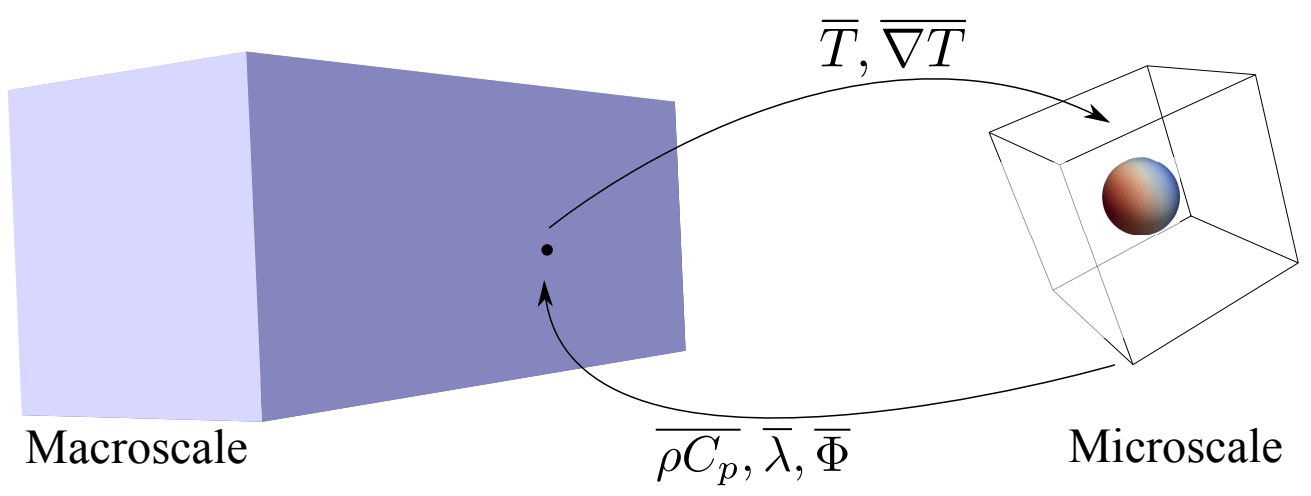

Figure 13: Scheme of the $\mathrm{FE}^{2}$ procedure.

and:

$$
\overline{\boldsymbol{\Phi}}=\frac{1}{|\Omega|} \int_{\Omega} \phi d \Omega .
$$

The effective tangent conductivity matrix is defined through:

$$
\bar{\lambda}=-\frac{\partial \overline{\mathbf{\Phi}}}{\partial \overline{\nabla T}}
$$

At each integration point of the macroscale discretization, $\bar{\lambda}$ can be evaluated numerically by finite difference approximation around the macroscopic temperature gradient $\overline{\nabla T}$ by:

$$
\bar{\lambda}_{i j}=-\frac{\bar{\Phi}_{i}\left(\overline{\nabla T}_{j}+\delta\right)-\bar{\Phi}_{i}\left(\overline{\nabla T}_{j}\right)}{\delta}
$$

where $\delta$ is a small numerical parameter chosen to be negligible as compared to $\overline{\nabla T}_{j}$. In $2 \mathrm{D}, \bar{\lambda}_{i j}$ requires three FEM computations on the RVE, and four FEM computations in 3D. In 2D for example, each of the three FEM computations corresponds to the RVE problem in which equation (31) is used with $\overline{\nabla T}, \overline{\nabla T}+\delta\left[\begin{array}{ll}1 & 0\end{array}\right]^{T}$ and $\overline{\nabla T}+\delta\left[\begin{array}{ll}0 & 1\end{array}\right]^{T}$ respectively. For the self-consistency of the paper, details on above relations are proposed in Appendix A.

\subsubsection{Macroscale equations}

The multiscale scheme is depicted in Fig. 13. At the macroscale, the model uses the effective quantities computed numerically from the RVE.

Considering a domain $\bar{\Omega} \subset \mathbb{R}^{D}$, where $D$ is the domain dimension with boundary $\partial \bar{\Omega}$, the problem to be solved at the macroscale is given by:

$$
\begin{gathered}
\overline{\rho C_{p}} \frac{\partial \bar{T}}{\partial t}-\nabla \cdot \bar{\Phi}+h\left(\bar{T}-T_{\infty}\right)=0 \text { in } \bar{\Omega}, \\
\bar{\Phi}=-\bar{\lambda} \overline{\nabla T} .
\end{gathered}
$$

where $\mathrm{h}$ is the convection coefficient and $T_{\infty}$ is the temperature of the media in contact with $\partial \bar{\Omega}_{h}$.

\subsubsection{FEM discretization and nonlinear solving procedure}

The weak form corresponding to (37) is given by

$$
\int_{\bar{\Omega}} \overline{\rho C_{p}} \frac{\partial \bar{T}}{\partial t} \delta \bar{T} d \Omega-\int_{\bar{\Omega}} \overline{\mathbf{\Phi}} \cdot \nabla \delta \bar{T} d \Omega+\int_{\partial \bar{\Omega}_{h}} h \bar{T} \delta \bar{T} d \Gamma_{h}-\int_{\partial \bar{\Omega}_{h}} h T_{\infty} \delta \bar{T} d \Gamma_{h}=0=R(\bar{T})
$$


where $\delta \bar{T}$ is a test function with sufficient regularity and satisfying $\delta \bar{T}=0$ on $\partial \Omega$. The above problem being highly nonlinear, a Newton solving procedure is proposed. Using Taylor expansion of $R$ around a known temperature solution $\bar{T}^{k}$, we have at the first order:

$$
\boldsymbol{R}\left(\bar{T}^{k}+\Delta \bar{T}\right)=\mathbf{R}\left(\bar{T}^{k}\right)+D_{\Delta \bar{T}} \mathbf{R}\left(\bar{T}^{k}\right) \Delta \bar{T}+O\left(\Delta \bar{T}^{2}\right)=0
$$

where $D_{\Delta \bar{T}} R\left(\bar{T}^{k}\right)$ is the directional derivative, defined by:

$$
D_{\Delta \bar{T}} R\left(\bar{T}^{k}\right)=\left[\frac{d R}{d \alpha}\left\{\bar{T}^{k}+\alpha \Delta \bar{T}\right\}\right]_{\alpha=0} .
$$

We have:

$$
\begin{gathered}
D_{\Delta \bar{T}}\left(\int_{\bar{\Omega}} \overline{\mathbf{\Phi}} \cdot \nabla \delta \bar{T} d \Omega+\int_{\partial \bar{\Omega}_{h}} h \bar{T} \delta \bar{T} d \Gamma\right)=\int_{\bar{\Omega}} D_{\Delta \bar{T}}\{\overline{\mathbf{\Phi}} \cdot \nabla \delta \bar{T}\} d \Omega+\int_{\partial \bar{\Omega}_{h}} h D_{\Delta \bar{T}}\{\bar{T}\} \delta \bar{T} d \Gamma \\
=\int_{\bar{\Omega}} \frac{\partial \overline{\mathbf{\Phi}}}{\partial \nabla \bar{T}} D_{\Delta \bar{T}}(\nabla \bar{T}) \cdot \nabla \delta \bar{T} d \Omega+\int_{\partial \bar{\Omega}_{h}} h \Delta \bar{T} \delta \bar{T} d \Gamma=-\int_{\bar{\Omega}} \bar{\lambda} \nabla(\Delta \bar{T}) \cdot \nabla \delta \bar{T} d \Omega+\int_{\partial \bar{\Omega}_{h}} h \Delta \bar{T} \delta \bar{T} d \Gamma .
\end{gathered}
$$

Introducing the following approximation for the time derivative:

$$
\frac{\partial \bar{T}}{\partial t} \simeq \frac{\bar{T}_{n+1}-\bar{T}_{n}}{\Delta t}
$$

then

$$
D_{\Delta \bar{T}}\left(\frac{\partial \bar{T}}{\partial t}\right) \simeq \frac{\Delta \bar{T}_{n+1}}{\Delta t} \equiv \frac{\Delta \bar{T}}{\Delta t} .
$$

Then:

$$
D_{\Delta \bar{T}}\left\{\int_{\bar{\Omega}} \overline{\rho C_{p}} \frac{\partial \bar{T}}{\partial t} \delta \bar{T} d \Omega\right\}=\int_{\bar{\Omega}} \overline{\rho C_{p}} \frac{\Delta \bar{T}}{\Delta t} \delta \bar{T} d \Omega .
$$

We finally obtain the linearized problem:

$$
\begin{aligned}
& \int_{\bar{\Omega}} \overline{\rho C_{p}} \frac{\Delta \bar{T}}{\Delta t} \delta \bar{T} d \Omega+\int_{\bar{\Omega}} \bar{\lambda} \nabla(\Delta \bar{T}) \cdot \nabla \delta \bar{T} d \Omega+\int_{\partial \bar{\Omega}_{h}} h \Delta \bar{T} \delta \bar{T} d \Gamma \\
& =\int_{\bar{\Omega}} \overline{\rho C_{p}} \frac{\left[\bar{T}_{n+1}^{k}-\bar{T}_{n}\right]}{\Delta t} \delta T d \Omega-\int_{\bar{\Omega}} \overline{\mathbf{\Phi}}\left(\bar{T}_{n+1}^{k}\right) \cdot \nabla(\delta \bar{T}) d \Omega+\int_{\partial \Omega_{h}}\left[\bar{T}_{n+1}^{k}-\bar{T}_{\infty}\right] \delta \bar{T} d \Gamma,
\end{aligned}
$$

that is solved to obtain $\Delta \bar{T}$. Then, the temperature at time $t_{n+1}$ is corrected until convergence of the norm of the residual is sufficiently small according to:

$$
\bar{T}_{n+1}^{k+1}=\bar{T}_{n+1}^{k}+\Delta \bar{T} .
$$

The macroscale structure is discretized using FEM elements. For the 2D examples, 3-nodes triangular elements are used while 4-nodes tetrahedral or 8-nodes hexaedral elements are used in 3D cases. In each element, the temperature increment and its gradient are approximated by:

$$
\Delta \bar{T}=\mathbf{N}(\mathbf{x}) \Delta \overline{\mathbf{T}}^{i}, \quad \nabla(\Delta \bar{T})=\mathbf{B}(\mathbf{x}) \Delta \overline{\mathbf{T}}^{i}
$$

where $\Delta \mathbf{T}^{i}$ denotes a vector of nodal values of $\Delta T$. The same discretization is used for the test function:

$$
\delta \bar{T}=\mathbf{N}(\mathbf{x}) \delta \overline{\mathbf{T}}^{i}, \quad \nabla(\delta \bar{T})=\mathbf{B}(\mathbf{x}) \delta \overline{\mathbf{T}}^{i},
$$


where $\mathbf{N}(\mathbf{x})$ and $\mathbf{B}(\mathbf{x})$ are vectors and matrices of shape functions, and of shape functions derivatives, respectively. Introducing the above discretization in (46) we obtain a linear system of equations in the form:

$$
\left(\frac{1}{\Delta t} \mathbf{C}+\mathbf{K}\right) \Delta \overline{\mathbf{T}}=-\mathbf{R},
$$

where

$$
\begin{gathered}
\mathbf{C}=\int_{\bar{\Omega}} \mathbf{N}^{T} \overline{\rho C_{p}}\left(\bar{T}_{n+1}^{k}\right) \mathbf{N} d \Omega \\
\mathbf{K}=\int_{\bar{\Omega}} \mathbf{B}^{T} \bar{\lambda}\left(\bar{T}_{n+1}^{k}\right) \mathbf{B} d \Omega+\int_{\partial \bar{\Omega}_{h}} \mathbf{N}^{T} h \mathbf{N} d \Gamma
\end{gathered}
$$

and

$$
\begin{gathered}
\mathbf{R}=\int_{\bar{\Omega}} \mathbf{N}^{T} \overline{\rho C_{p}} \\
\left.\bar{T}_{n+1}^{k}\right) \mathbf{N}\left(\left[\overline{\mathbf{T}}_{n+1}^{k}\right]^{i}-\overline{\mathbf{T}}_{n}^{i}\right) d \Omega-\int_{\bar{\Omega}} \mathbf{B}^{T} \overline{\mathbf{\Phi}}\left(\bar{T}_{n+1}^{k}\right) d \Omega \\
+\int_{\partial \bar{\Omega}_{h}} h \mathbf{N}^{T}\left(\mathbf{N}\left[\overline{\mathbf{T}}_{n+1}^{k}\right]^{i}-T_{\infty}\right) d \Gamma .
\end{gathered}
$$

Then, when the transient nonlinear thermal regime is considered, the overall algorithm is described as follows:

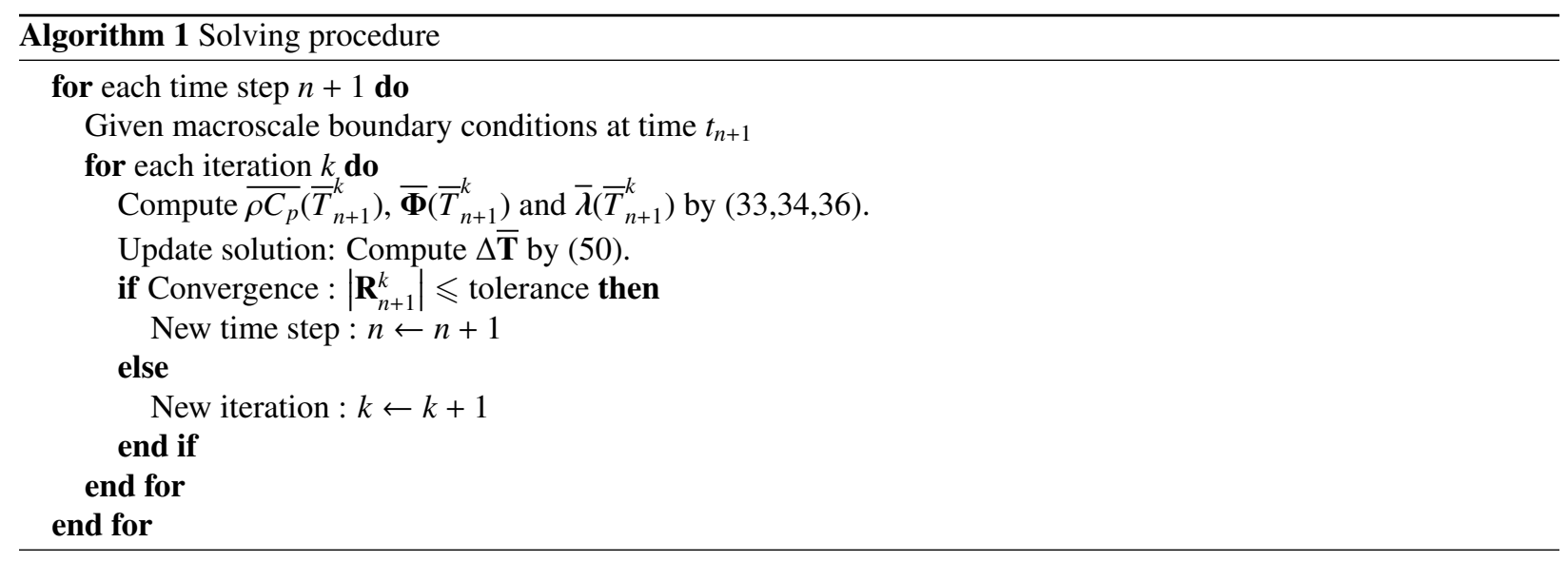

If the steady-state regime is considered for the thermal problem, the above linear system reduces to:

$$
\mathbf{K} \Delta \overline{\mathbf{T}}=-\mathbf{R}^{\prime},
$$

with

$$
\mathbf{R}^{\prime}=-\int_{\bar{\Omega}} \mathbf{B}^{T} \overline{\mathbf{\Phi}}\left(\bar{T}_{n+1}^{k}\right) d \Omega+\int_{\partial \bar{\Omega}_{h}} h \mathbf{N}^{T}\left(\mathbf{N}\left[\overline{\mathbf{T}}_{n+1}^{k}\right]^{i}-T_{\infty}\right) d \Gamma .
$$

\section{Numerical examples}

\subsection{Stationary heat transfer}

We first validate the present procedure in a steady-state regime at both scales. The geometry of the structure is depicted in Fig. 14.

The RVEs used in 2D and 3D are depicted in Fig. 15. The radius of the PCM inclusions is taken as $R=0.2 \mathrm{~mm}$ and the thickness of the membrane is taken as $e=0.015 \mathrm{~mm}$. The thermo-physical properties of the matrix, of the membrane and of the PCM phases are listed in table 2. The PCM latent heat of fusion is taken as $L_{F}=1.68 \times 10^{5}$ 


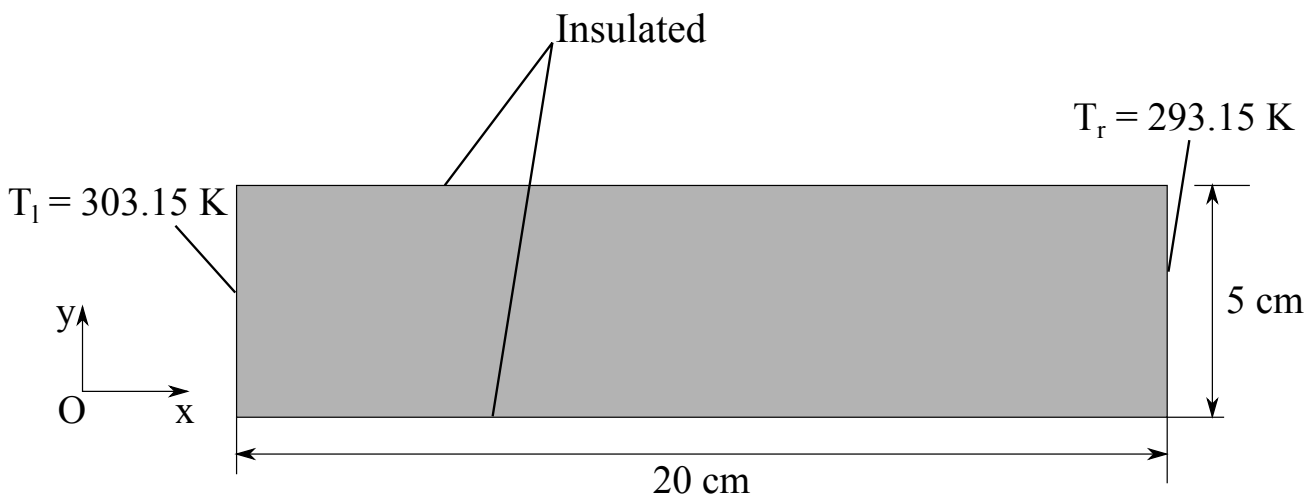

Figure 14: 2D steady state problem: geometry of the macroscale structure.

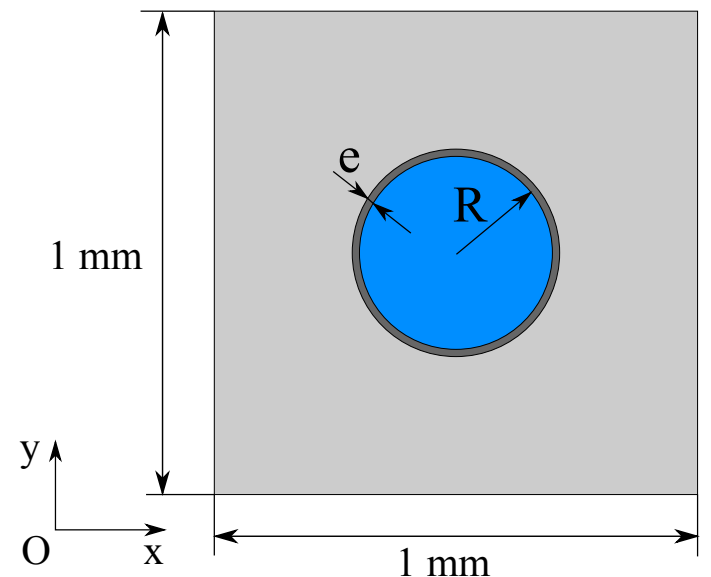

(a)

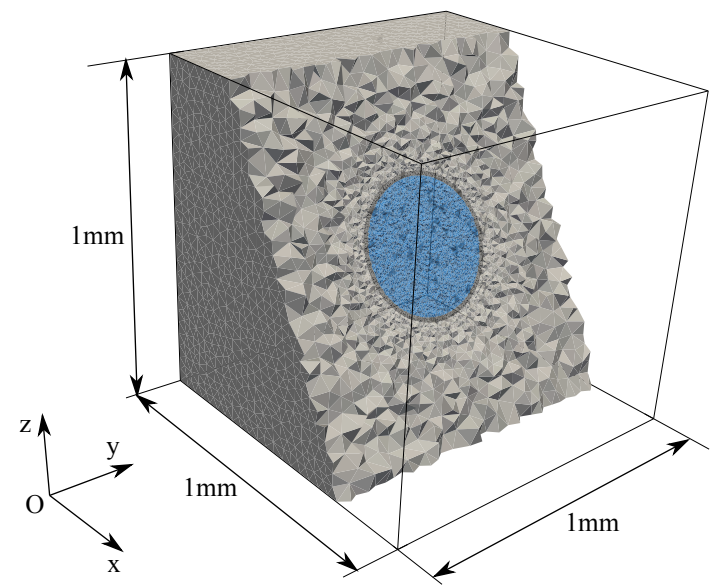

(b)

Figure 15: RVE: geometry; (a) 2D RVE; (b) 3D RVE. 
Table 2: Thermo-physical proprieties of the phase within the RVE.

\begin{tabular}{lccc}
\hline & $\lambda\left[\mathrm{W} . \mathrm{m}^{-1} \cdot \mathrm{K}^{-1}\right]$ & $\rho\left[\mathrm{kg} . \mathrm{m}^{-3}\right]$ & $C_{p}\left[\mathrm{~J} . \mathrm{kg}^{-1}\right]$ \\
\hline Matrix & 0.92 & 2300 & 880 \\
\hline Membrane & 0.17 & 1200 & 1400 \\
\hline Solid PCM & 0.23 & 900 & 2400 \\
\hline Liquid PCM & 0.15 & 934 & 1800 \\
\hline
\end{tabular}

J.kg ${ }^{-1}$ with a melting point at $300.15 \mathrm{~K}\left(T_{s}=299.65 \mathrm{~K}\right.$ and $\left.T_{\ell}=300.65 \mathrm{~K}\right)$. The dynamic viscosity of the liquid phase is $\eta_{\ell}=3.42 \times 10^{-3}$ Pa.s.

At the macroscale, the mesh is composed of 512 triangular elements. The RVE is discretized with 2518 triangular elements. Constant temperatures are applied on the macroscale structure boundaries: $T_{l}=303.15 \mathrm{~K}$ at $x=0 \mathrm{~cm}$ and $T_{r}=293.15 \mathrm{~K}$ at $x=20 \mathrm{~cm}$ (see Fig. 14). On the other boundaries, the heat flux is zero.

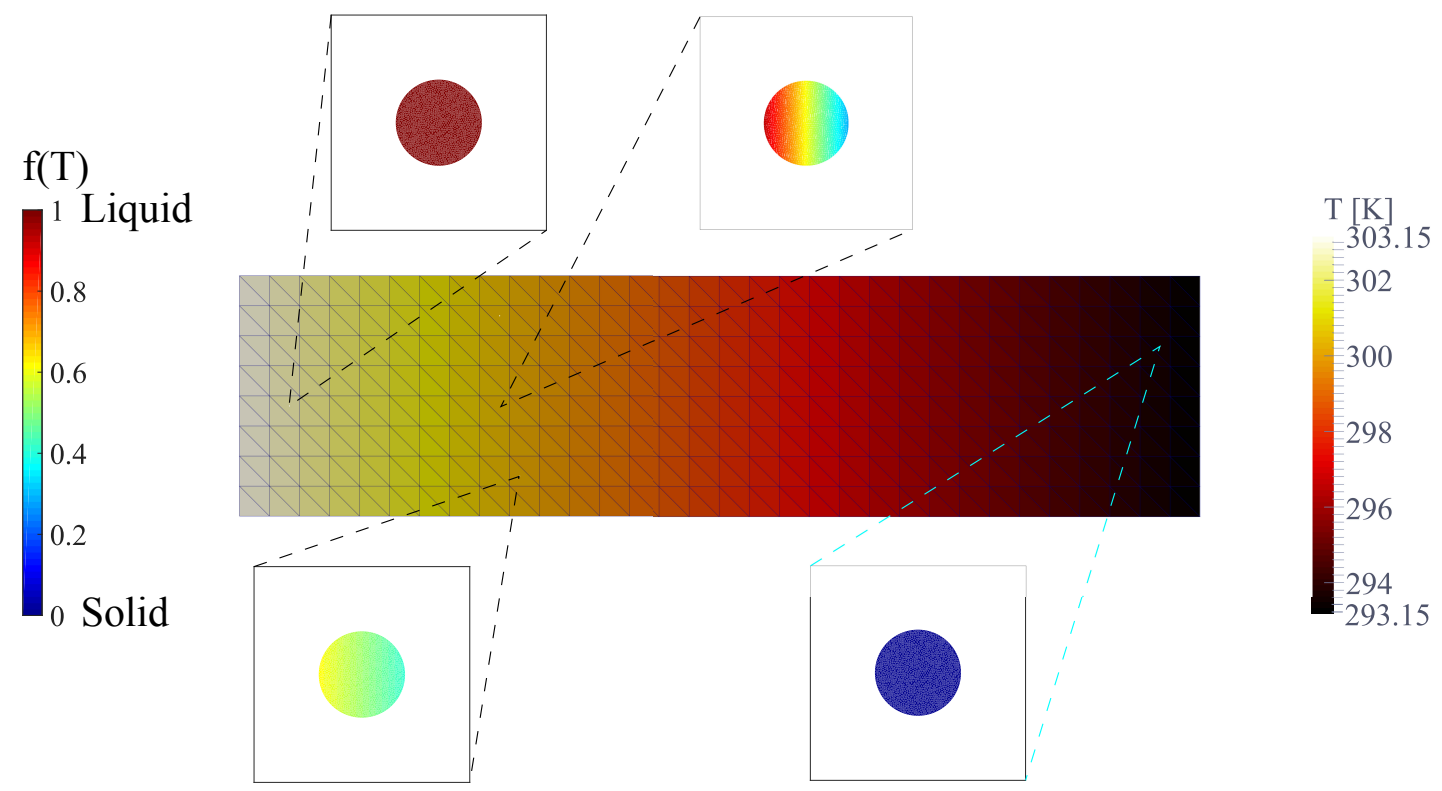

Figure 16: Two scales steady-state solution for the 2D case.

Fig. 16 shows the temperature solution at the macroscale and the liquid phase indicator $f(T)$ within different RVEs. The indicator $f(T)$ takes 0 value for solid phase and 1 for liquid phase. In regions of the structure where the macroscopic temperature field is close to the melting point of the PCM, we can notice incomplete melted PCM in the corresponding RVEs. To validate the $\mathrm{FE}^{2}$ model, a complete FEM analysis is performed where all heterogeneities are explicitly described (see Fig. 17). The heterogeneous structure is composed of periodically distributed cells identical to the RVE of Fig. 15a.

In this case, the constant temperatures $T=303.15 \mathrm{~K}$ and $T=297.15 \mathrm{~K}$ are prescribed on the left and right ends. The macroscale mesh is composed of 40 triangular elements. For the full FEM analysis, the mesh is composed of 406768 triangular elements.

Fig. 18 shows the $x$-component of the heat flux and the phase indicator in the PCM inclusions for the full FEM analysis. The temperature at $y=0.5 \mathrm{~mm}$ is compared to the $\mathrm{FE}^{2}$ solution (see Fig. 19). We can note a good agreement, even though the $\mathrm{FE}^{2}$ solution cannot describe the small fluctuations due to the PCM particles, as expected. To compare the heat fluxes, we compute the mean value of $\bar{\Phi}_{x}$ along the $y$-axis for the full FEM solution as a function of $x$ : 


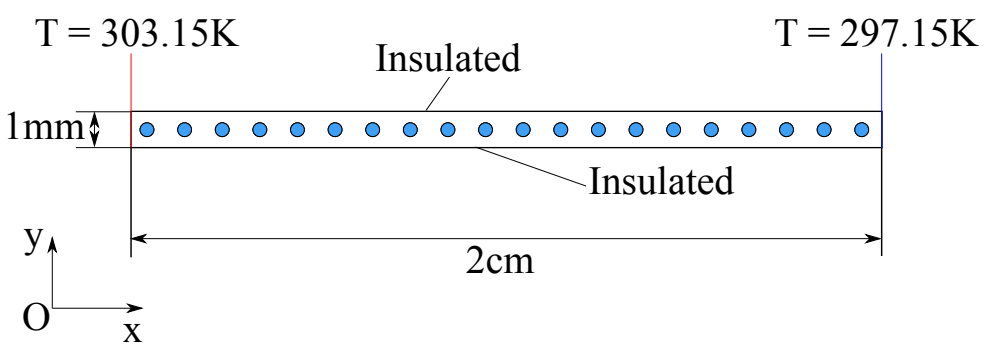

Figure 17: Structure used for the validation of the $\mathrm{FE}^{2}$ procedure.
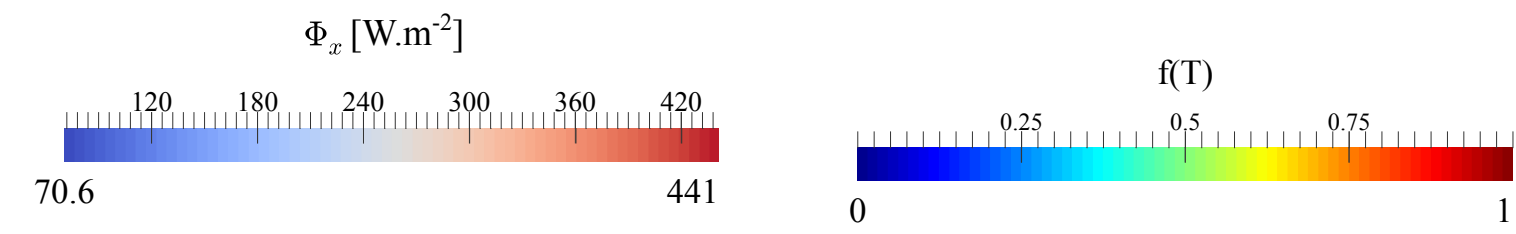

(a)

(b)

Figure 18: Full FEM analysis: (a) $x$-component of the heat flux; (b) phase indicator.

$$
\bar{\Phi}_{x}(x)=\frac{1}{y_{\max }-y_{\min }} \int_{y_{\min }}^{y_{\max }} \phi_{x} d y .
$$

For the $\mathrm{FE}^{2}$ model, the $x$-component of the flux does not vary along the $y$ axis. In Fig. 19, the $x$-component of the heat flux obtained from $\mathrm{FE}^{2}$ is compared to the mean value expressed by Eq. (56), showing a good agreement on the average values. As the microscale temperature fluctuations are averaged out, we observe a small gap between the curves, especially around the melting temperature. However, the zoom box in 19a shows that this gap remains negligible.

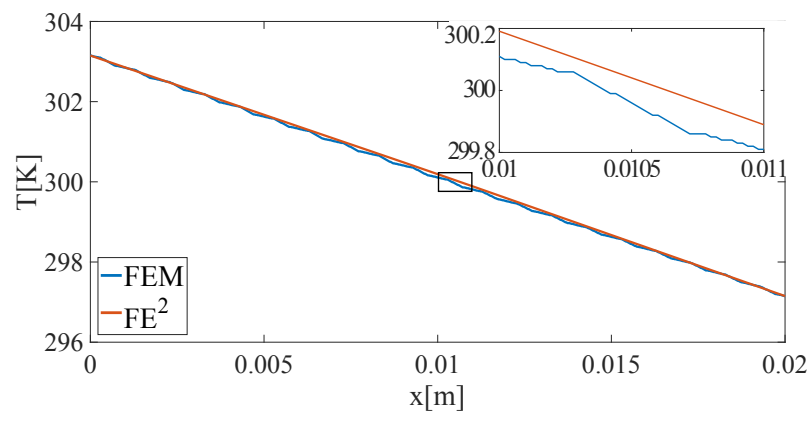

(a)

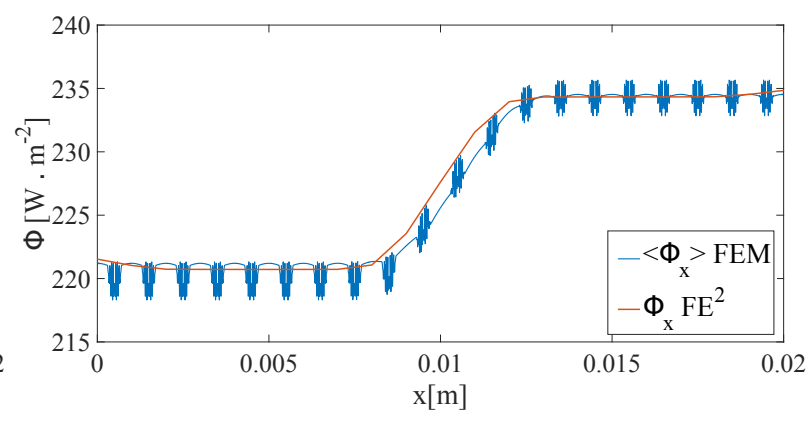

(b)

Figure 19: Comparison between $\mathrm{FEM}$ and $\mathrm{FE}^{2}$ results along $x$-axis: (a) temperature field; (b) heat fluxes.

The computational gains between the direct solving and the $\mathrm{FE}^{2}$ are (54\% reduction). However, the reference problem of Fig. 18 is of small size to allow its resolution in a reasonable time. For this reason, the computational gains between the direct solving and the $\mathrm{FE}^{2}$ are not drastic. However, these gains dramatically increase with the size of the mesh and especially in 3D where the direct solving is simply not feasible (see section 5.3). To better show the micro-scale phase changes effects, the transient regime is studied in the following. 


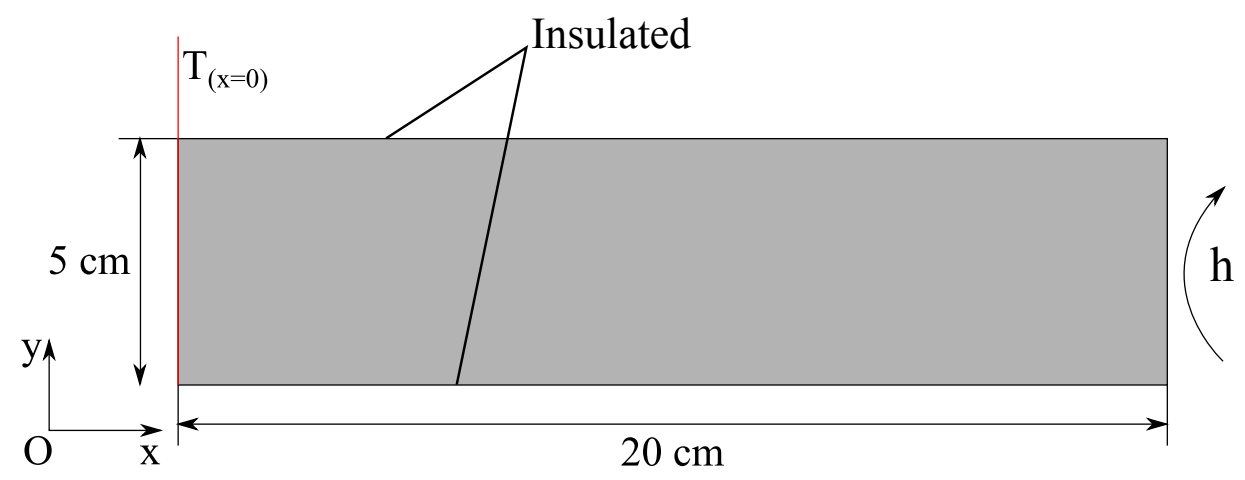

Figure 20: Geometry and boundary conditions for the 2D transient thermal problem.

\section{2. $2 D$ transient thermal conduction}

In this example, we consider transient thermal conduction regime at the macroscopic scale. Choosing large time steps at the macroscale yields steady state regime at the microscale due to scale separation. For this analysis, we consider the case depicted in Fig. 20. The thermal conductivity of the matrix is $1.7 \mathrm{~W} \cdot \mathrm{m}^{-1} \cdot \mathrm{K}^{-1}$. The macroscale time step is $\Delta t=60 \mathrm{~s}$. Time-dependent boundary conditions are prescribed at $x=0 \mathrm{~cm}$ by:

$$
T_{(x=0)}=T_{0}+\delta T \sin \left(\frac{2 \pi t}{t_{F}}\right) .
$$

A convective exchange is considered at the right-end of the structure, with $h=10 \mathrm{~W} \cdot \mathrm{m}^{-2} \cdot \mathrm{K}^{-1}$ and $T_{\infty}=297.15$ $\mathrm{K}$. The other boundaries are insulated. In (57), $t$ is the current time, $T_{0}=300.15 \mathrm{~K}, \delta T=10 \mathrm{~K}$ and $t_{F}$ is the total simulation time fixed at 12 hours. $T_{(x=0)}$ is a sine function which varies up to $\pm 5 K$ around the melting temperature of the PCM every 3 hours (see Fig. 21). The initial temperature is considered as $T_{i}=300.15 \mathrm{~K}$.

Fig. 21 shows the temperature applied at $x=0 \mathrm{~cm}$ and the temperature at different locations of the macroscale structure with and without PCM. These curves show a significant offset between the inner temperature of the structure embedding PCM and the structure without PCM capsules. The temperature peaks are smoother in the structure with PCM. There is an offset up to $4 \mathrm{~K}$ compared to the homogeneous structure. This confirms the experimental results reported in several works of the literature [25], and demonstrates the capacity of the PCM capsules to regulate the temperature in structures and buildings.

\section{3. $3 D$ transient thermal conduction}

We now consider the 3D case shown in Fig. 22. The thermo-physical properties of the RVE are the same as in Table 2. The RVE used is shown in Fig. 15b. The radius of the PCM capsule is $R=0.3 \mathrm{~mm}$ (corresponding to a volume fraction of 0.113 ). Initially, the whole structure is at $T_{i}=297.15 \mathrm{~K}$. A constant temperature of $303.15 \mathrm{~K}$ is applied at $x=0 \mathrm{~cm}$ and $T=297.15 \mathrm{~K}$ at $x=20 \mathrm{~cm}$, while the rest of the boundary is insulated. The final time simulation is 4 hours. The macroscale time step is $\Delta t=60 \mathrm{~s}$. The structure is discretized with 6144 tetrahedral elements while the RVE is composed of 36315 tetrahedral elements. Fig. 23 shows the macroscale temperature solution and the liquid phase indicators on four specific RVEs at $t=4$ hours.

As expected, at $x=5 \mathrm{~cm}$, as the macroscale temperature is higher than the melting point, the PCM is fully melted. In the same way, there is a fully solid PCM in the RVE at $x=19.17 \mathrm{~cm}$ because the macroscale temperature is less than the melting temperature of PCM. On the other hand, there are partially melted PCM in the RVEs where the macroscale temperature is close to the melting point of the PCM. Fig. 24 shows the temperature at the center cross line $(y=5 \mathrm{~cm}, z=5 \mathrm{~cm})$ for different times, and the temperature evolution with time at $x=10 \mathrm{~cm}, y=5 \mathrm{~cm}, z=5$ $\mathrm{cm}$, with and without PCM.

\section{4. $3 D$ thermo-mechanical example}

In this next example, we apply the method by taking into account the thermo-mechanical effects. We consider the 3D macroscale structure shown in Fig. 25. The geometry is discretized with 2795 8-node hexahedra. A constant 


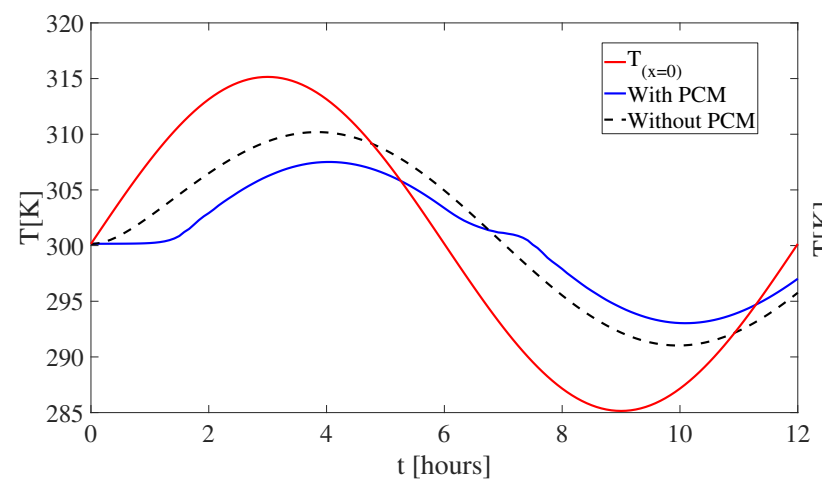

(a) $x=5 \mathrm{~cm}, y=2.5 \mathrm{~cm}$

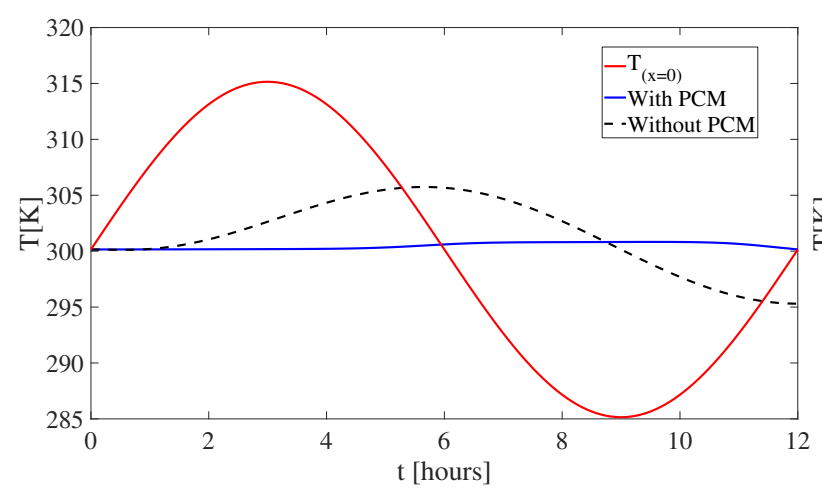

(c) $x=15 \mathrm{~cm}, y=2.5 \mathrm{~cm}$

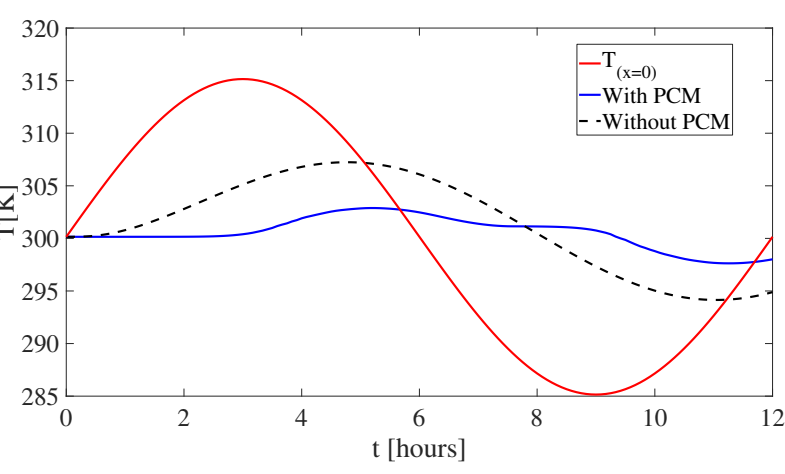

(b) $x=10 \mathrm{~cm}, y=2.5 \mathrm{~cm}$

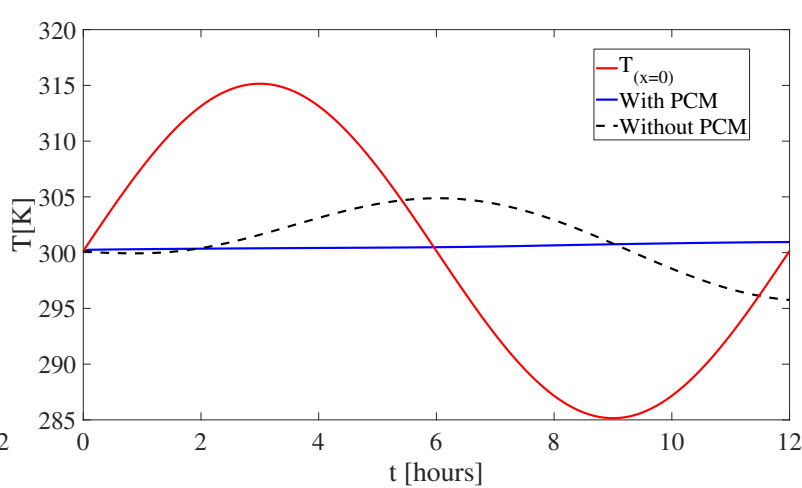

(d) $x=20 \mathrm{~cm}, y=2.5 \mathrm{~cm}$

Figure 21: Temperature at different points of the macroscopic structure.

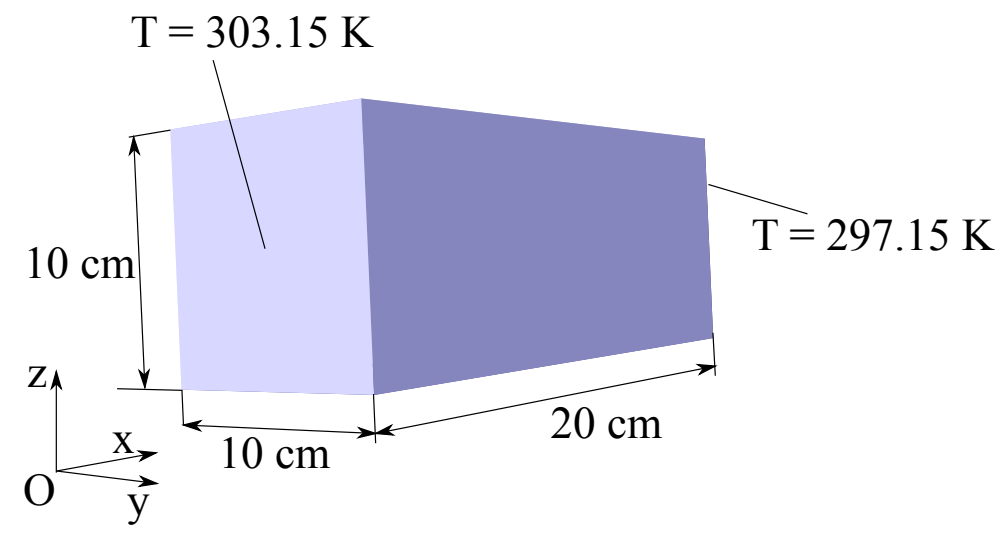

Figure 22: Maroscopic scale structure for the 3D thermal problem. 


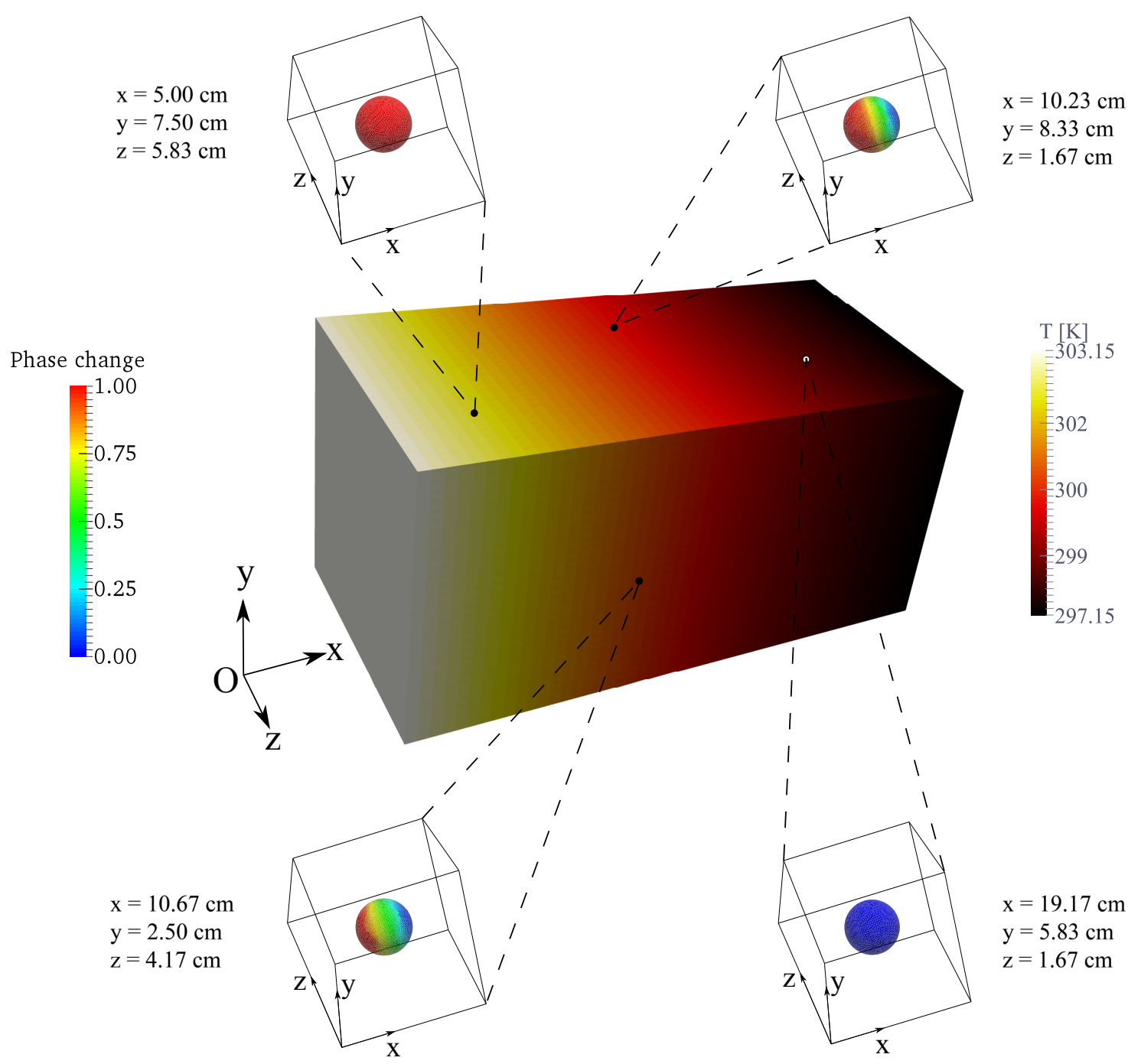

Figure 23: Two scales temperature solution at $t=4 \mathrm{~h}$.

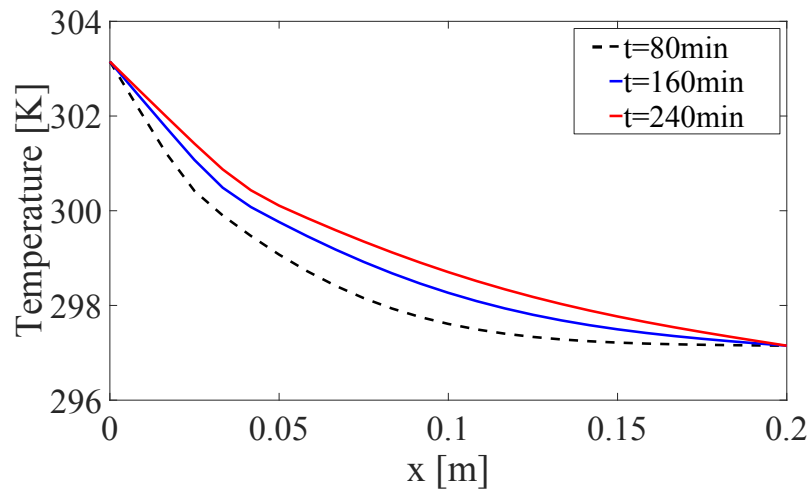

(a) Temperature at $y=5 \mathrm{~cm}, z=5 \mathrm{~cm}$.

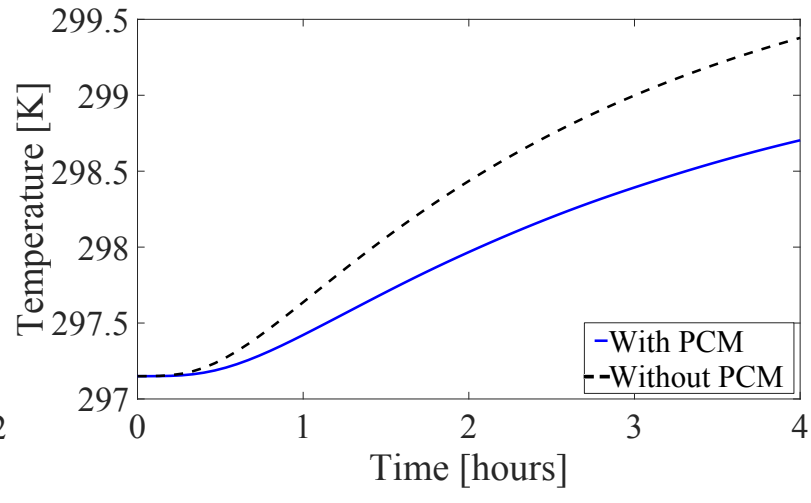

(b) Temperature at $x=10 \mathrm{~cm}, y=5 \mathrm{~cm}, z=5 \mathrm{~cm}$.

Figure 24: Temperature in the structure at the macro scale: (a) temperature along the $x$-axis for different times; (b) temperature at the center of the structure with and without PCM particles. 


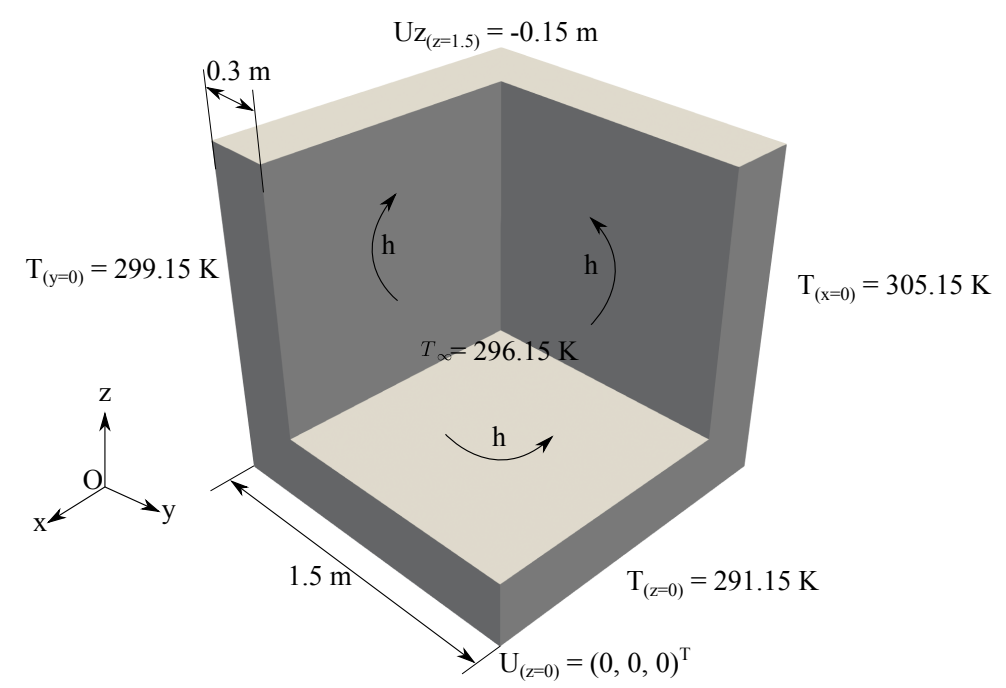

Figure 25: 3D thermo mechanical example: geometry and boundary conditions.

temperatures of $305.15 \mathrm{~K}, 299.15 \mathrm{~K}$ and $291.15 \mathrm{~K}$ are respectively applied on the surfaces at $x=0, y=0$ and $z=0$. A convection heat flux is considered at three surfaces and the others are insulated, as described in Fig. 25. The mechanical load is as follows: the bottom side of the structure $(z=0)$ is blocked and $z$-components of the displacement at the upper side are set to $-0.15 \mathrm{~m}$. The material properties are listed in Tables 1 and 2 . The thermal conductivity of the matrix is $1.7 \mathrm{~W} . \mathrm{m}^{-1} \cdot \mathrm{K}^{-1}$ and the radius of PCM is $0.3 \mathrm{~mm}$.

Fig. 26 shows the thermal fields within the macroscopic structure. At the side parallel to the plan $x=0$, we can observe that the temperature gradient is high, resulting in an important heat flux. Close to the bottom, the heat flux decreases and far from this location, the heat flux is almost one-dimensional.

The mechanical fields are depicted in Fig. 27: the displacement magnitude and the mean stress (von Mises). The macroscale solution has been obtained using the effective stiffness matrix, considering the PCM at solid state, as it has been shown in section 3 that the phase change does not affect significantly the effective elastic tensor if the stiffness of the matrix is high. This example shows the potential of the present framework to handle more realistic industrial cases of thermomechanical simulation of buildings embedding micro-encapsulated PCM.

\subsection{Effects of PCM volume fraction}

In this example, the volume fraction of PCM is varied to study its effects on the macroscopic heat transfer. The case presented in Fig. 20 is performed for five values of $\mathrm{R}: 100 \mu \mathrm{m}, 150 \mu \mathrm{m}, 200 \mu \mathrm{m}, 250 \mu \mathrm{m}$ and $300 \mu \mathrm{m}$. A constant temperature of $T=313.15 \mathrm{~K}$ is applied at $x=0 \mathrm{~cm}$.

As expected, the volume fraction of microscale inclusion has an effect on the macroscale heat conduction. In Fig. 28, the temperature profile in the structure increases when the radius of the PCM capsule decreases. We can observe a kink in the curves which corresponds to the position of the melting front. Due to the phase change, we have two different macroscale thermal behaviour at each side of the kink. The offset between the zone with liquid PCM and the zone with solid PCM increases with the PCM volume fraction which explains why the kink in the curves is more pronounced for $28.27 \%$ of PCM. In Fig. 29b, we compare the offset of the temperature between the homogeneous structure and the PCM composite, using data from Fig. 29a: this offset increases with the radius of PCM. This curve shows the effectiveness of the composite compared to the matrix alone. As the heat flux passes through the matrix, these results can be improved by using higher thermal conductivity of the PCM (see e.g. [26]).

\section{Conclusion}

In this study, a $\mathrm{FE}^{2}$ multilevel method has been developed to study the heat transfer and the mechanical behavior in structures made of concrete based composites embedding PCMs in both 2D and 3D cases. An equivalent capacity 


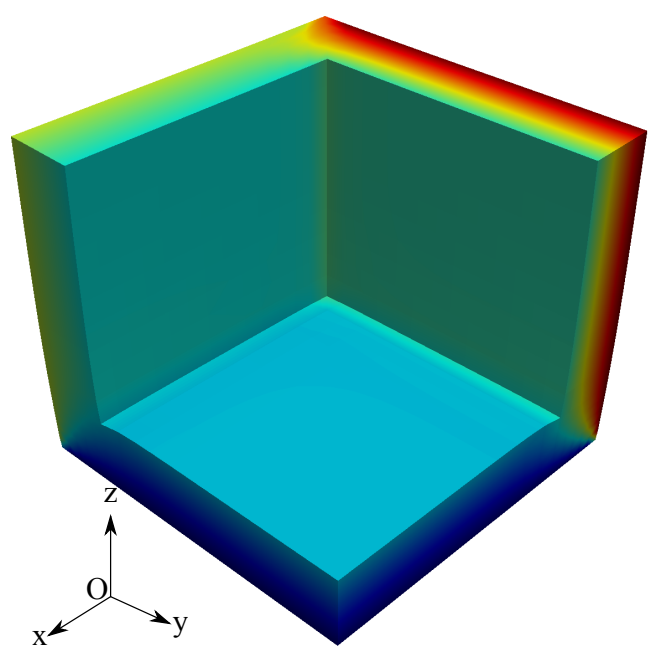

(a)

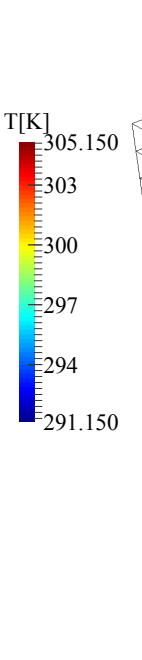

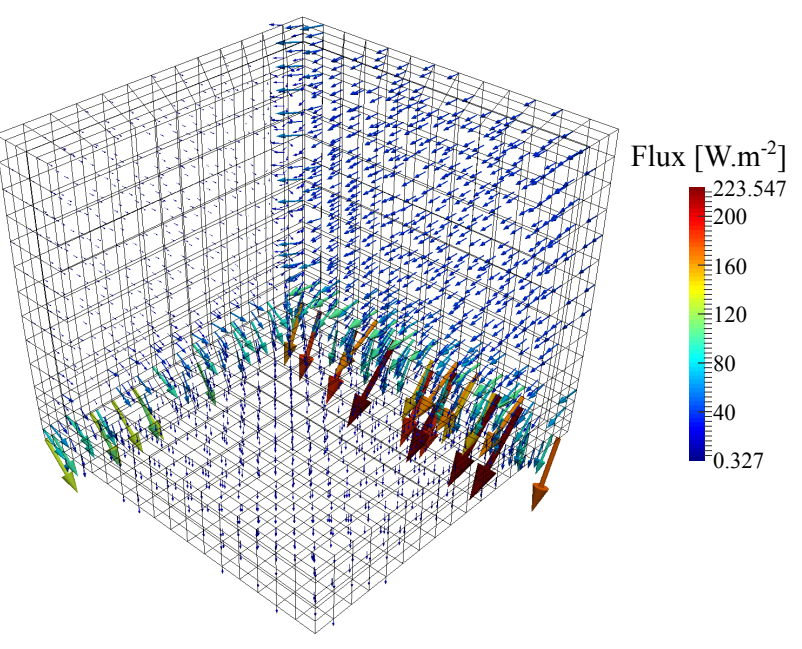

(b)

Figure 26: Thermal fields: (a) temperature; (b) heat flux.

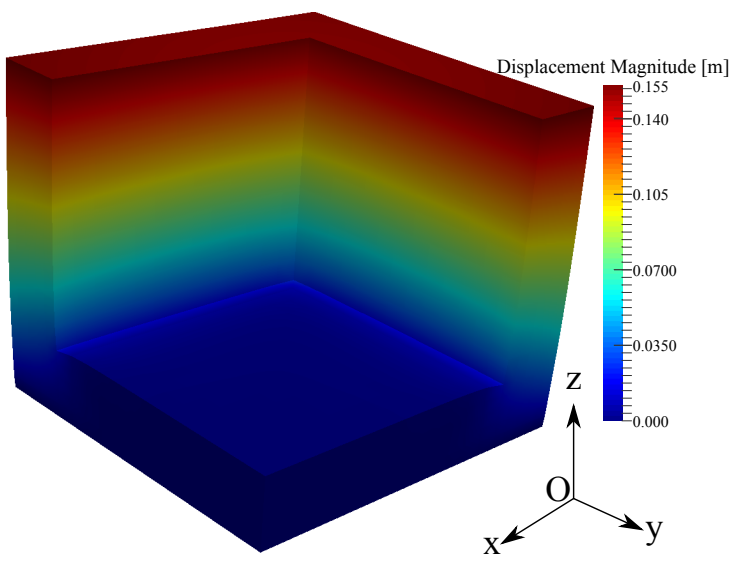

(a)

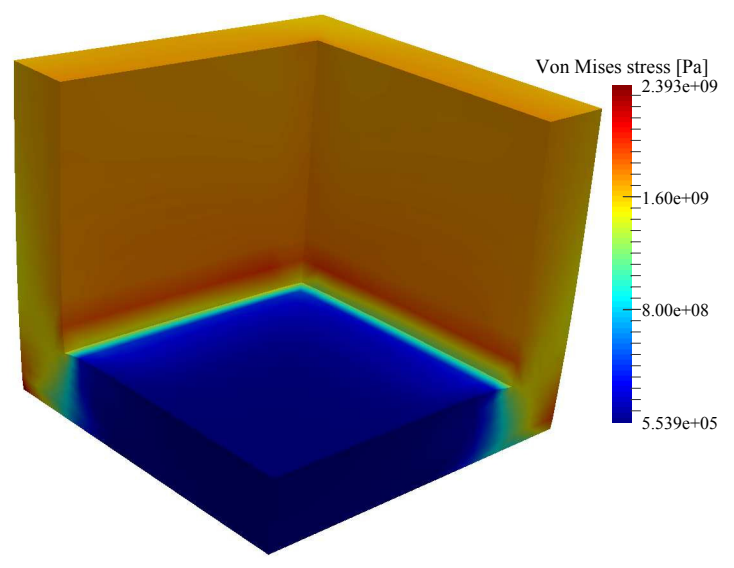

(b)

Figure 27: Mechanical fields: (a) displacements; (b) von Mises stress.

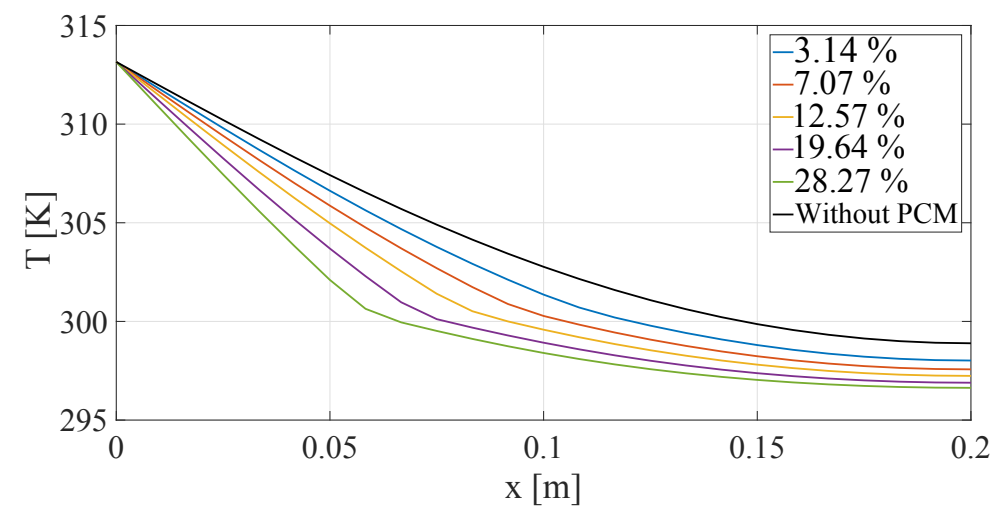

Figure 28: Temperature along x-axis $(y=2.5 \mathrm{~cm})$ for different volume fraction of PCM at $t=4$ hours. 


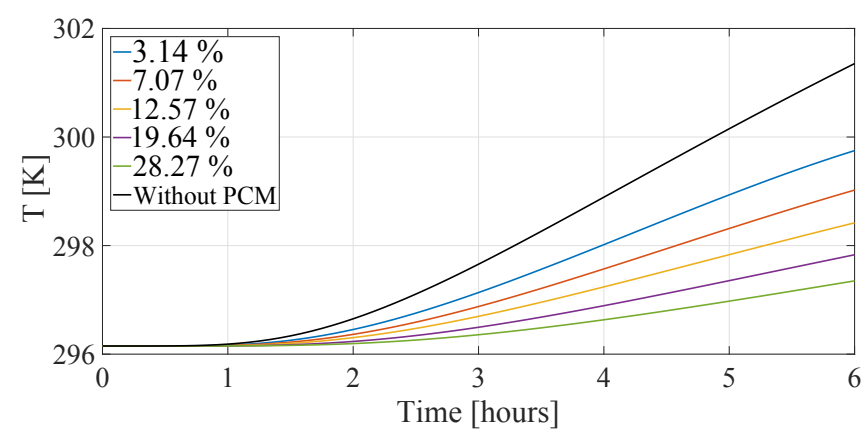

(a)

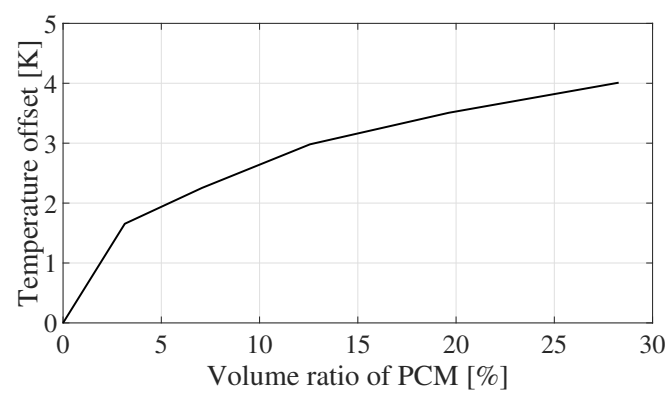

(b)

Figure 29: Effects of volume fraction of PCM: (a) Temperature at $(x=20 \mathrm{~cm}, y=2.5 \mathrm{~cm})$ with time. (b) Offset between the temperature in the structure without PCM and the structures with different volume ratio of PCM at $(x=20 \mathrm{~cm}, y=2.5 \mathrm{~cm})$ after 6 hours.

method has been used to model thermal phase changes within the PCM. The developed numerical framework allows studying the strong nonlinear, history-dependent thermal response of the structure embedding PCM, which is not feasible if all heterogeneities are explicitly meshed due to the scale separation between the size of the structure and of the dimensions of the PCM capsules. The study shows the benefits of PCM on the effective thermal behavior: the peaks of temperature in the structure are smoothed and shifted as compared to the material without PCM. We have shown that the offset between the temperature in the structure and the applied temperature increases with the radius of the PCM's capsule in the RVE. It has also been shown that due to the large stiffness of the matrix, the effects of the thermal problem over the mechanical response are negligible. The trends obtained by the numerical results are in good agreement with the experimental observations.

\section{Acknowledgments}

The authors gratefully acknowledge the financial support from LABEX Multi-Scale Modelling \& Experimentation of Materials for Sustainable Construction (MMCD), through ANR Investments for the Future program ANR-11LABX-022-01. Pr Julien Yvonnet aknowledges the financial support from Institut Universitaire de France (IUF).

\section{Appendix A. Scale bridging relations}

\section{Appendix A.1. Average of temperature gradient}

We consider a 2D RVE composed of two phases (see Fig. A.30). The two phases occupy respectively two domains $\Omega^{1}$ and $\Omega^{2}$ which are separated by an interface $\Gamma$.

$\boldsymbol{n}$ is the outward unit vector at the boundary. The temperature fields in each domain are respectively denoted by $T^{1}$ and $T^{2}$. Using the divergence theorem, the volume average of temperature gradient in the microscale domain $\Omega=\Omega^{1} \cup \Omega^{2}$ is given by:

$$
\begin{aligned}
\frac{1}{|\Omega|} \int_{\Omega} \nabla T d \Omega & =\frac{1}{|\Omega|} \int_{\partial \Omega} T^{1} \boldsymbol{n} d \Gamma+\frac{1}{|\Omega|} \int_{\Gamma} T^{1} n^{1} d \Gamma+\frac{1}{|\Omega|} \int_{\Gamma} T^{2} \boldsymbol{n}^{2} d \Gamma \\
& =\frac{1}{|\Omega|} \int_{\partial \Omega} T^{1} \boldsymbol{n} d \Gamma+\frac{1}{|\Omega|} \int_{\Gamma}\left(T^{1}-T^{2}\right) \boldsymbol{n}^{1} d \Gamma .
\end{aligned}
$$

Assuming perfect interfaces, $T^{1}=T^{2}$ across $\Gamma$. Using (29) we have

$$
\frac{1}{|\Omega|} \int_{\Omega} \nabla T d \Omega=\frac{1}{|\Omega|} \int_{\partial \Omega} T^{1} \boldsymbol{n} d \Gamma=\frac{1}{|\Omega|} \int_{\partial \Omega} \overline{\nabla T} \boldsymbol{x} \cdot \boldsymbol{n} d \Gamma .
$$

Applying the divergence theorem again on the last term of (A.2) and noting that $\nabla \boldsymbol{x}=\mathbf{1}$, we finally obtain 


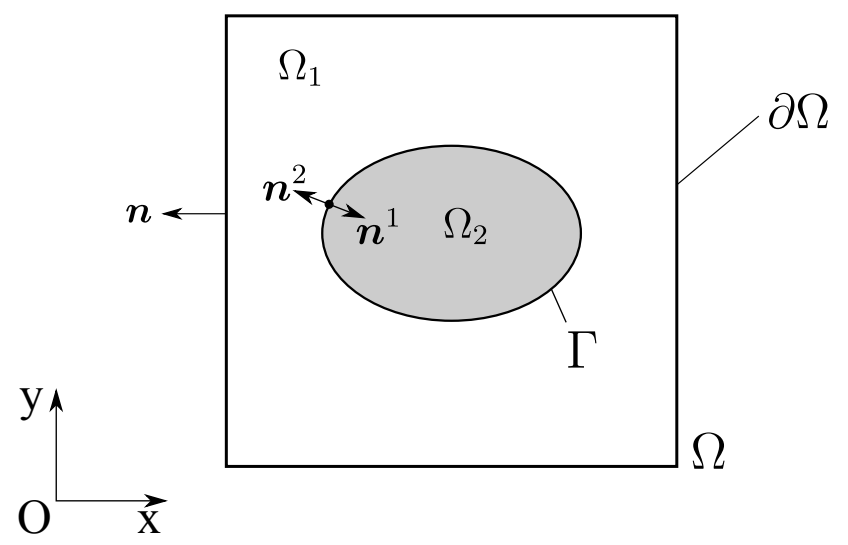

Figure A.30: Two phases RVE.

$$
\frac{1}{|\Omega|} \int_{\Omega} \nabla T d \Omega=\left(\frac{1}{|\Omega|} \int_{\Omega} 1 d \Omega_{m}\right) \overline{\nabla T}=\overline{\nabla T}
$$

Appendix A.2. Average of heat flux

In this section, we give the proof of (34) which express the equivalence between the volume average of the microscale heat flux and the macroscale heat flux. Noting that $\phi_{i, i}=0$, we have:

$$
\frac{\partial}{\partial x_{i}}\left(\phi_{i} x_{j}\right)=\left(\phi_{i} x_{j}\right)_{, i}=\phi_{i, i} x_{j}+\phi_{i}\left(x_{j}\right)_{, i}=\phi_{i} \delta_{i j}=\phi_{j}
$$

From (A.4) and using the divergence theorem,

$$
\frac{1}{|\Omega|} \int_{\Omega} \phi_{j} d \Omega=\frac{1}{|\Omega|} \int_{\Omega}\left(\phi_{i} x_{j}\right)_{, i} d \Omega=\frac{1}{|\Omega|} \int_{\partial \Omega} \phi_{i} n_{i} x_{j} d \Gamma .
$$

Considering the RVE depicted in Fig. A.30, $\phi^{1}$ and $\phi^{2}$ denote the heat flux respectively in $\Omega^{1}$ and $\Omega^{2}$. Then,

$$
\begin{gathered}
\frac{1}{|\Omega|} \int_{\Omega} \phi_{j} d \Omega=\frac{1}{|\Omega|} \int_{\Omega^{1}} \phi_{j}^{1} d \Omega+\frac{1}{|\Omega|} \int_{\Omega^{2}} \phi_{j}^{2} d \Omega \\
=\frac{1}{|\Omega|} \int_{\partial \Omega} \phi_{i}^{1} n_{i} x_{j} d \Gamma+\frac{1}{|\Omega|} \int_{\Gamma} \phi_{i}^{1} n_{i}^{1} x_{j} d \Gamma-\frac{1}{|\Omega|} \int_{\Gamma} \phi_{i}^{2} n_{i}^{1} x_{j} d \Gamma
\end{gathered}
$$

Assuming perfect interface, $\phi^{1}=\phi^{2}$ across $\Gamma$. (A.6) becomes

$$
\frac{1}{|\Omega|} \int_{\Omega} \phi_{j} d \Omega=\frac{1}{|\Omega|} \int_{\partial \Omega} \phi_{i}^{1} n_{i} x_{j} d \Gamma
$$

Using uniform heat flux boundary conditions expressed as

$$
\overline{\boldsymbol{\Phi}} \cdot \boldsymbol{n}=\boldsymbol{\phi} \cdot \boldsymbol{n} \text { on } \partial \Omega
$$

we have:

$$
\frac{1}{|\Omega|} \int_{\Omega} \phi_{j} d \Omega=\frac{1}{|\Omega|} \int_{\partial \Omega} \bar{\Phi}_{i} n_{i} x_{j} d \Gamma=\frac{1}{|\Omega|} \int_{\Omega}\left(\bar{\Phi}_{i} x_{j}\right)_{, i} d \Omega=\bar{\Phi}_{j}
$$




\section{References}

[1] M. Karkri, M. Lachheb, F. Albouchi, S. B. Nasrallah, I. Krupa, Thermal properties of smart microencapsulated paraffin/plaster composites for the thermal regulation of buildings, Energy and Buildings 88 (2015) 183-192.

[2] D. Hawes, D. Banu, D. Feldman, The stability of phase change materials in concrete, Solar Energy Materials and Solar Cells 27 (2) (1992) 103 - 118, special Issue on Heat Storage Materials.

[3] D. Hawes, D. Feldman, Special issue on heat storage materials absorption of phase change materials in concrete, Solar Energy Materials and Solar Cells 27 (2) (1992) $91-101$.

[4] K. Ismail, C. Alves, M. Modesto, Numerical and experimental study on the solidification of PCM around a vertical axially finned isothermal cylinder, Applied Thermal Engineering 21 (1) (2001) $53-77$.

[5] X. Wang, Y. Zhang, W. Xiao, R. Zeng, Q. Zhang, H. Di, Review on thermal performance of phase change energy storage building envelope, Chinese Science Bulletin 54 (6) (2009) 920-928.

[6] I. O. Salyer, A. K. Sircar, Phase change katerials for heating and cooling of residential buildings and other applications, Proceedings of the 25th Intersociety Energy Conversion Engineering Conference 4 (4) (1990) 236-243.

[7] D. Snoeck, B. Priem, P. Dubruel, N. De Belie, Encapsulated phase-change materials as additives in cementitious materials to promote thermal comfort in concrete constructions, Materials and Structures 49 (1) (2016) 225-239.

[8] Y. Tian, C. Zhao, A numerical investigation of heat transfer in phase change materials (PCMs) embedded in porous metals, Energy 36 (9) (2011) 5539-5546.

[9] S. F. Hosseinizadeh, A. A. R. Darzi, F. Tan, Numerical investigations of unconstrained melting of nano-enhanced phase change material (NEPCM) inside a spherical container, International Journal of Thermal Sciences 51 (2012) 77-83.

[10] M. Aguayo, S. Das, C. Castro, N. Kabay, G. Sant, N. Neithalath, Porous inclusions as hosts for phase change materials in cementitious composites: Characterization, thermal performance, and analytical models, Construction and Building Materials 134 (2017) 574 - 584.

[11] T. Mori, K. Tanaka, Average stress in matrix and average elastic energy of materials with misfitting inclusions, Acta Metallurgica 21 (5) (1973) $571-574$

[12] J. Renard, M. Marmonier, Etude de l'initiation de l'endommagement dans la matrice d'un matériau composite par une méthode d'homogénéisation, Aerospace Science and Technology 9 (1987) 36-51.

[13] F. Feyel, Multiscale $\mathrm{FE}^{2}$ elastoviscoplastic analysis of composite structure, Comput. Mater. Sci. 16(1-4) (1999) $433-454$

[14] K. Terada, N. Kikuchi, A class of general algorithms for multi-scale analysis of heterogeneous media, Comput. Meth. Appl. Mech. Eng. 190 (2001) 5427-5464.

[15] V. Kouznetsova, M. G. D. Geers, W. A. M. Brekelmans, Multi-scale constitutive modelling of heterogeneous materials with a gradientenhanced computational homogenization scheme, International Journal for Numerical Methods in Engineering 54 (8) (2002) 1235-1260.

[16] M. Geers, J. Yvonnet, Multiscale modeling of microstructure-property relations, MRS Bull. 41(08) (2016) 610-616.

[17] I. Özdemir, W. A. M. Brekelmans, M. G. D. Geers, Computational homogenization for heat conduction in heterogeneous solids, International Journal for Numerical Methods in Engineering 73 (2) (2008) 185-204.

[18] M. Kabir, L. Chernova, M. Bartsch, Numerical investigation of room-temperature deformation behavior of a duplex type $\gamma$ tial alloy using a multi-scale modeling approach, Acta Materialia 58 (17) (2010) 5834 - 5847.

[19] M. Aadmi, M. Karkri, M. E. Hammouti, Heat transfer characteristics of thermal energy storage of a composite phase change materials: Numerical and experimental investigations, Energy 72 (2014) $381-392$

[20] A. Joulin, Z. Younsi, L. Zalewski, S. Lassue, D. R. Rousse, J.-P. Cavrot, Experimental and numerical investigation of a phase change material: Thermal energy storage and release, Applied Energy 88 (7) (2011) $2454-2462$.

[21] R. Viswanath, Y. Jaluria, A comparison of different solution methodologies for melting and solidification problems in enclosures, Numerical Heat Transfer, Part B: Fundamentals 24 (1) (1993) 77-105.

[22] B. Rubinsky, E. G. Cravahlo, A finite element method for the solution of one-dimensional phase change problems, International Journal of Heat and Mass Transfer 24 (12) (1981) 1987 - 1989.

[23] Y. Ding, J. A. Gear, K. N. Tran, A finite element modeling of thermal conductivity of fabrics embedded with phase change material, in: Proceedings of the 8th Biennial Engineering Mathematics and Applications Conference, EMAC-2007, Vol. 49 of ANZIAM J., 2008, pp. C439-C456.

[24] U. Küttler, W. A. Wall, Fixed-point fluid-structure interaction solvers with dynamic relaxation, Computational Mechanics 43 (1) (2008) 61-72.

[25] M. Kenisarin, K. Mahkamov, Solar energy storage using phase change materials, Renewable and Sustainable Energy Reviews 11 (9) (2007) $1913-1965$.

[26] Y. Zhong, Q. Guo, S. Li, J. Shi, L. Liu, Heat transfer enhancement of paraffin wax using graphite foam for thermal energy storage, Solar Energy Materials and Solar Cells 94 (6) (2010) 1011 - 1014. 